\title{
Analysis of Centranthera grandiflora Benth Transcriptome Explores Genes of Catalpol, Acteoside and Azafrin Biosynthesis
}

\author{
Xiaodong Zhang ${ }^{1,2,+}$, Caixia $\mathrm{Li}^{1,2, \dagger}$, Lianchun Wang ${ }^{1}$, Yahong Fei ${ }^{3}$ and Wensheng Qin ${ }^{4, *}$ \\ 1 College of Chemistry Biology and Environment, Yuxi Normal University, Yuxi 653100, China; \\ zxd95@yxnu.edu.cn (X.Z.); lcx@yxnu.edu.cn (C.L.); wanglianchun@yxnu.edu.cn (L.W.) \\ 2 Food and Bioengineering College, Xuchang University, Xuchang 461000, China \\ 3 Yuxi Flyingbear Agricultural Development Company Limited, Yuxi 653100, China; feiyahong@gmail.com \\ 4 Department of Biology, Lakehead University, Thunder Bay, ON P7B 5E1, Canada \\ * Correspondence: wqin@lakeheadu.ca; Tel.: +1-807-343-8467 \\ + These authors contribute to this article equally.
}

Received: 14 September 2019; Accepted: 27 November 2019; Published: 29 November 2019

\begin{abstract}
Cardiovascular diseases (CVDs) are a major cause of health loss in the world. Prevention and treatment of this disease by traditional Chinese medicine is a promising method. Centranthera grandiflora Benth is a high-value medicinal herb in the prevention and treatment of CVDs; its main medicinal components include iridoid glycosides, phenylethanoid glycosides, and azafrin in roots. However, biosynthetic pathways of these components and their regulatory mechanisms are unknown. Furthermore, there are no genomic resources of this herb. In this article, we provide sequence and transcript abundance data for the root, stem, and leaf transcriptome of $C$. grandiflora Benth obtained by the Illumina Hiseq2000. More than 438 million clean reads were obtained from root, stem, and leaf libraries, which produced 153,198 unigenes. Based on databases annotation, a total of 557, 213, and 161 unigenes were annotated to catalpol, acteoside, and azafrin biosynthetic pathways, respectively. Differentially expressed gene analysis identified 14,875 unigenes differentially enriched between leaf and root with 8,054 upregulated genes and 6,821 downregulated genes. Candidate MYB transcription factors involved in catalpol, acteoside, and azafrin biosynthesis were also predicated. This work is the first transcriptome analysis in C. grandiflora Benth which will aid the deciphering of biosynthesis pathways and regulatory mechanisms of active components.
\end{abstract}

Keywords: Centranthera grandiflora Benth; transcriptome; catalpol biosynthesis; acteoside biosynthesis; azafrin biosynthesis

\section{Introduction}

Cardiovascular diseases (CVDs) are an important reason for death in the world which hinder sustainable development of human beings [1]. In China, CVDs were also the leading cause of death due to lifestyle changes, urbanization, and the accelerated process of aging, and the figures have exceeded $42 \%$ of all deaths in both rural and urban regions, which was much higher than deaths caused by cancer or any other diseases in 2014 [2]. Traditional Chinese medicine has been used for more than 2000 years and has displayed the explicit role in preventing and treating CVDs, although the detailed pharmacological mechanisms have seldomly been clarified [3]. Centranthera grandiflora Benth, also known as broad bean Ganoderma lucidum, wild broad bean root, Huaxuedan, Golden Cat's Head, and Xiaohongyao, is a medicinal plant widely used for preventing and treating CVDs among Miao Nationality of Yunnan in China. In taxonomy, it belongs to the Centranthera, Scrophulariaceae family. Distinguished as a rare and endangered medicinal plant, C. grandiflora Benth usually grows 
well with Cyperus rotundus and is mainly distributed in Yunnan, Guizhou, and Guangxi in China as well as parts of India, Myanmar, and Vietnam [4-7]. Its roots possess many functions, such as to promote blood circulation, to regulate menstruation, to dispel blood stasis, and to relieve pain, and has known coagulation, antibacterial, and anticancer properties [6,8-10]. Therefore, it is mainly used to treat amenorrhea, dysmenorrhea, metrorrhagia, fall-related injuries, rheumatic bone pain, traumatic hemorrhage, and cardiovascular and cerebrovascular diseases [6,8-10].

So far, studies on this herb have mainly focused on the isolation and identification of its chemical constituents and pharmacological effects, while the discovery of genes related to biosynthesis of active secondary metabolites has not been reported. Azafrin and D-mannitol were first isolated and identified from the roots of C. grandiflora Benth in 1984 [8]. Then, aeginetin and azalea were isolated from the roots of $C$. grandiflora Benth, and their coagulation, antimicrobial, and anticancer functions were verified in 2012 [10]. In the same year, nine iridoid glycosides including aucubin, mussaenoside, 8-epiloganin, 8-epiloganic acid, mussaenosidic acid, catalpol, gardoside methyl ester, geniposidic acid, and 6-O-methylaucubin were isolated from roots of $C$. grandiflora Benth [6]. In 2014, another 17 compounds, including six new ones: centrantheroside $\mathrm{A}$ to $\mathrm{E}$ and neomelasmoside; phenylethanoid glycosides: plantainoside $\mathrm{A}$, calceolarioside $\mathrm{A}$, acteoside, and isoacteoside; monoterpenoid glycosides: melasmoside and rehmaionoside C; Di-O-methylcrenatin; azafrin; $\beta$-sitosterol; mannitol; and $\beta$-daucosterol were isolated from $C$. grandiflora Benth roots [5,7]. Studies have shown that iridoid glycosides, phenylethanoid glycosides, and azafrin are the main substance bases for their pharmacodynamics [5,7]. In 2017, tissue culture of C. grandiflora Benth was also successfully developed [11].

At present, C. grandiflora Benth roots sold in public markets are mainly collected from wild resources, while its artificial cultivation has just started [5]. So far, the cost of annual C. grandiflora Benth planting is about $\$ 0.13$ million per hectare and the worth of annual yield is about $\$ 0.64$ million per hectare [5]. Therefore, to explore the biosynthetic pathways and regulatory mechanisms of the main active ingredients of $C$. grandiflora Benth will lay a scientific foundation for breeding new varieties of this herb and for producing its medicinal chemical constituents by synthetic biology.

Iridoid glycosides belong to monoterpenoids, and their biosynthesis in plants can be divided into three stages. The first stage is precursor formation, which includes the plastidial 2-C-methyl-D-erythritol-4-phosphate (MEP) pathway and the cytoplasmic mevalonate (MVA) pathway to produce isopentenyl diphosphate (IPP) and dimethylallyl diphosphate (DMAPP) [12,13]. The second stage is the formation of a carbon skeleton structure [13-16]. The third stage is the post-modification of terpenoids: hydroxylation, methylation, isomerization, demethylation, glycosylation, etc. [16]. So far, most of the biosynthesis pathways of iridoid glycosides remain unclear. However, the complete catalpol biosynthetic pathway was first elicited in Picrorhiza kurroa [17], and it was partially decoded in Rehmannia glutinosa [18]. In P. kurroa, the catalpol biosynthetic pathway contains 29 steps including 14 steps for the MEP and MVA pathways and 15 steps for the iridoid pathway [17]. As the MEP and MVA pathways has been widely and intensively studied and they are conserved in plants [19], here, we mainly focused on the iridoid pathway. So far, two iridoid pathways including secoiridoid pathway (Route I) and decarboxylated iridoid pathway (Route II) have been reported, and the early enzymatic steps containing geranyl diphosphate synthase (GPPS), geraniol synthase (GES), geraniol 10-hydroxylase (G10H), 8-hydroxygeraniol oxidoreductase (8HGO), iridoid synthase (IS), iridoid oxidase (IO), and UDP-glucosyltransferase (UGT) are common to both pathways [20], has been verified in Catharanthus roseus and P. kurroa [14,21,22], and proposed in Gardenia jasminoides [23,24]. The remaining steps were first deduced by chemical intermediates [20], and then the corresponding enzymes were predicted and discovered by transcriptome analysis [17]. In P. kurroa, another seven enzymes containing aldehyde dehydrogenase (ALD), flavanone 3-dioxygenase/hydoxylase (F3D), 2-hydroxyisoflavanone dehydratase (2FHD), deacetoxycephalosporin-C hydroxylase (DCH), uroporphyrinogen decarboxylase/UDP-glucuronic acid decarboxylase (UPD/UGD), and squalene 
monooxygenase (SQM) have been proposed to catalyze the remaining seven steps in catalpol biosynthesis [17].

Acteoside, belonging to phenylethanoid glycosides, is composed of two parts: caffeoyl CoA and hydroxytyrosol glucoside [25]. Feeding and inhibition experiments showed that hydroxytyrosol glucoside moiety is derived from tyrosine while caffeoyl CoA moiety is derived from phenylalanine via the cinnamate pathway and that both tyrosine and phenylalanine come from the shikimate pathway [26,27]. In Ole europae and R. glutinosa, phenylalanine is converted into caffeoyl CoA via four enzymes including phenylalanine ammonia-lyase (PAL), cinnamate-4-hydroxylase $(\mathrm{C} 4 \mathrm{H})$, coumarate-3-hydroxylase (C3H), and 4-coumarate-CoA ligase (4CL) $[18,25,28]$. Simultaneously, tyrosine is transformed into hydroxytyrosol glucoside through two alternative pathways: one is via $L$-dopa, dopamine, and hydroxytyrosol with the enzymes polyphenol oxidase (PPO), tyrosine decarboxylase (TDC), copper-containing amine oxidase (CuAO), alcohol dehydrogenase (ADH), and UGT; the other is via tyramine, tyrosol, and salidroside with the enzymes TDC, $\mathrm{CuAO}, \mathrm{ADH}, \mathrm{UGT}$, and PPO $[18,25,28]$. Finally, caffeoyl CoA and hydroxytyrosol glucoside can be converted into acteoside by Shikimate O-hydroxycinnamoyltransferase (HCT) and UGT [18,25]. Recent studies have verified that acteoside possesses pharmacological properties: antioxidant, anti-inflammatory, antidepressant, antitumor, antidiabetes, and hepatoprotection [29-32].

Azafrin, belonging to carotenoid derivative, is one of the most abundant active ingredients in C. grandiflora Benth roots and plays an important role in myocardial protection [33]. Carotenoids are ubiquitous pigments in plants, and they confer plants with bright yellows, oranges, and reds [34]. In higher plants, carotenoids are synthesized through isoprene-like pathways in plastids, including condensation, dehydrogenation, cyclization, hydroxylation, and epoxidation reactions, while lycopene acts as an important branch point of both synthesis of $\alpha$-carotene and $\beta$-carotene [35]. In the $\alpha$-carotene pathway, $\alpha$-carotene is synthesized by lycopene $\varepsilon$-cyclase (LCY- $\varepsilon$ ) and lycopene $\beta$-cyclase (LCY- $\beta$ ) and is then converted to lutein by $\varepsilon$-hydroxylase (LUT1) and $\beta$-cyclohexylase (LUT5) $[17,18]$. In the $\beta$-carotene pathway, LCY- $\beta$ catalyzes the synthesis of $\beta$-carotene, which can be converted into strigolactone, astaxanthin, capsanthin, capsorubin, and violaxanthin under the catalysis of different enzymes, while violaxanthin can be further converted into abscisic acid [36,37]. However, studies have shown that azafrin is an apocarotenoid which is generated by cleavage of carotenoids at the $\mathrm{C}^{\prime}-\mathrm{C} 10^{\prime}[38,39]$. In the strigolactone pathway, $\beta$-carotene is converted into carlactone through 9-cis-carotene, $10^{\prime}$-apo- $\beta$-carotenal by enzymes DWARF27, carotenoid cleavage dioxygenase 7 (CCD7), and CCD8 [39]. The intermediate product $10^{\prime}$-apo- $\beta$-carotenal is very similar to azafrin in structure except one terminal carboxyl group and two hydroxyl groups. Therefore, the hypothesis that azafrin is synthesized via $10^{\prime}$-apo- $\beta$-carotenal is proposed in this article.

Thus, the aim of this research is to characterize globally for the first time the transcriptomes of the root, stem, and leaf of $C$. grandiflora Benth using the Illumina Hiseq2000. To explore the genes involved in the catalpol, acteoside, and azafrin biosynthesis pathways and regulatory mechanisms, transcripts from leaves, stems, and roots of C. grandiflora Benth were screened out, quantified, and annotated. The results obtained here will facilitate further molecular studies in C. grandiflora Benth.

\section{Results}

\subsection{Sequencing and Assembly}

To figure out which genes are involved in the biosynthesis of active components in C. grandiflora Benth, nine sequencing libraries including roots (C_R1, C_R2, and C_R3), stems (C_S1, C_S2, and C_S3), and leaves (C_L1, C_L2, and C_L3) were prepared and sequenced with the Illumina Hiseq2000 platform. As a result, more than 45 million clean reads per library were obtained after cleaning and quality examination. Quality assessments of the sequencing data are shown in Table 1 . The error rate of all libraries was $0.03 \%$, while Q20 and Q30 were over $94.99 \%$ and $88.32 \%$, respectively, indicating that 
these data are suitable for further analysis. The raw data from the nine libraries have been deposited into the Short Reads Archive (SRA) database under the accession numbers: SRX6654843-SRX6654851.

Table 1. Quality assessment of the sequencing data.

\begin{tabular}{cccccccc}
\hline Sample & Raw Reads & Clean Reads & Clean Bases & Error (\%) & Q20 (\%) & Q30 (\%) & GC (\%) \\
\hline C_R1 & 49187308 & 48514118 & $7.28 G$ & 0.03 & 95.57 & 89.01 & 47.36 \\
C_R2 & 49633082 & 48674546 & $7.3 G$ & 0.03 & 97.80 & 93.85 & 49.17 \\
C_R3 & 48837996 & 47783570 & $7.17 G$ & 0.03 & 97.94 & 94.13 & 48.63 \\
C_S1 & 55750138 & 55002348 & $8.25 G$ & 0.03 & 95.53 & 89.08 & 47.82 \\
C_S2 & 49324046 & 48163930 & $7.22 \mathrm{G}$ & 0.03 & 97.47 & 93.33 & 49.39 \\
C_S3 & 50001598 & 48875538 & $7.33 G$ & 0.03 & 97.63 & 93.61 & 49.65 \\
C_L1 & 50405504 & 49123820 & $7.37 G$ & 0.03 & 95.66 & 89.19 & 48.69 \\
C_L2 & 45678578 & 45075310 & 6.76G & 0.03 & 97.59 & 93.45 & 49.89 \\
C_L3 & 47606624 & 46899750 & 7.03G & 0.03 & 94.99 & 88.32 & 49.60 \\
\hline
\end{tabular}

Note: C_R1, C_R2, and C_R3: three root samples; C_S1,C_S2, and C_S3: three stem samples; and C_L1, C_L2, and C_L3: three leaf samples.

The clean reads were combined and assembled by Trinity with min_kmer_cov set to 2 and all other default parameters [40]. Assembled sequences were subjected to cluster using the Trinity algorithm. As a result, 153,300 contigs were clustered into 173,851 trinity components. Each Trinity component contained a set of transcripts derived from the same gene, and a unigene was designated as the longest transcript in each trinity component. A total of 173,851 transcripts and 153,198 genes were assembled, with 69,421 (39.93\%) transcripts and 69,419 (45.31\%) genes being over $2 \mathrm{~Kb}$ in length (Figure 1). The average length of transcripts and genes were $1895 \mathrm{bp}$ and $2115 \mathrm{bp}$, respectively (Table 2), and the N50 for transcripts and genes were 2902 and 2936 bp, respectively (Table 2).

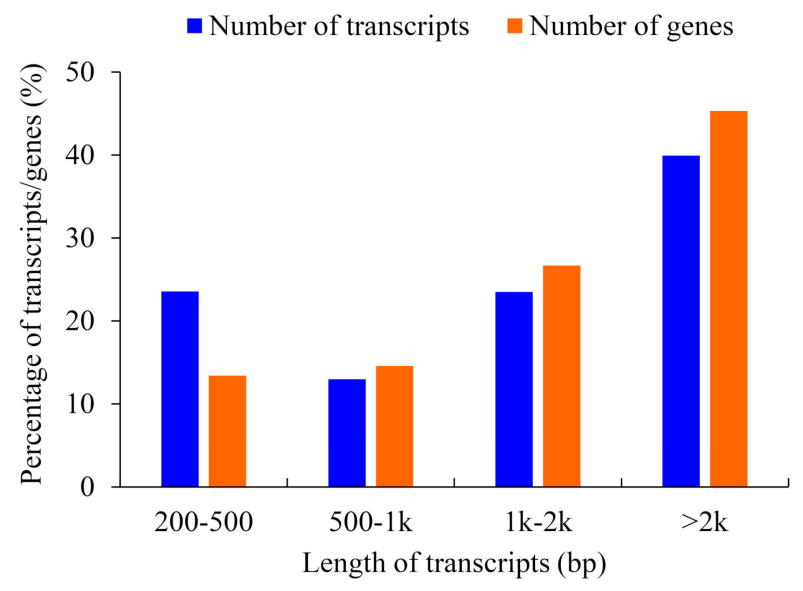

Figure 1. Length distribution frequency of spliced transcripts and deduced genes.

Table 2. Length frequency distribution of the spliced transcripts and genes.

\begin{tabular}{cccccccc}
\hline & $\begin{array}{c}\text { Min } \\
\text { Length }\end{array}$ & $\begin{array}{c}\text { Mean } \\
\text { Length }\end{array}$ & $\begin{array}{c}\text { Median } \\
\text { Length }\end{array}$ & $\begin{array}{c}\text { Max } \\
\text { Length }\end{array}$ & N50 & N90 & $\begin{array}{c}\text { Total } \\
\text { Nucleotides }\end{array}$ \\
\hline Transcripts & 201 & 1895 & 1574 & 16,816 & 2902 & 1089 & $329,518,919$ \\
Genes & 201 & 2115 & 1824 & 16,816 & 2936 & 1195 & $323,991,974$ \\
\hline
\end{tabular}

\subsection{Gene Function Annotation and Classification}

All the 153,198 assembled putative unigenes were aligned using the BLAST (Basic Local Alignment Search Tool) program against the seven classic databases including NR (nonredundant protein sequences), NT (Nucleotide collection), PFAM (Protein family), SwissProt, KOG (euKaryotic Orthologous Groups), KEGG (Kyoto Encyclopedia of Genes and Genomes), and GO (Gene Ontology) 
databases with $e$-value cutoffs of $10^{-5}, 10^{-5}, 10^{-2}, 10^{-5}, 10^{-3}, 10^{-10}$, and $10^{-6}$, respectively. A total of 26,652 unigenes $(17.39 \%)$ were annotated to the above seven databases in common, while 132,896 unigenes $(86.74 \%)$ were annotated in at least one database (Table 3). Among them, 127,767 unigenes $(83.39 \%)$ showed high similarity with sequences in the NR database ( $e$-value $\left.=10^{-5}\right), 96,216$ unigenes $(62.80 \%)$ matched to protein sequences in NT, and 103,257 unigenes $(67.40 \%)$ showed homology with known genes in SwissProt. The detailed results are shown in Table 3 and Tables S1-S3. Based on the top-hit species distribution of the homology results against NR database, the highest matches were genes from Sesamum indicum (43.77\%), followed by Handroanthus impetiginosus (22.58\%) and Erythranthe guttata (13.07\%) (Figure 2).

Table 3. Statistical results of gene annotation.

\begin{tabular}{ccc}
\hline Item & Number of Unigenes (n) & Percentage (\%) \\
\hline Annotated in NR & 127,767 & 83.39 \\
Annotated in NT & 96,216 & 62.80 \\
Annotated in SwissProt & 103,257 & 67.40 \\
Annotated in PFAM & 98,364 & 64.20 \\
Annotated in GO & 98,364 & 64.20 \\
Annotated in KOG & 44,170 & 28.83 \\
Annotated in KEGG & 57,190 & 37.33 \\
Annotated in all Databases & 26,652 & 17.39 \\
Annotated in at least one Database & 132,896 & 86.74 \\
Total unigenes & 153,198 & 100 \\
\hline
\end{tabular}

Note: NR (nonredundant protein sequences), NT (Nucleotide collection), PFAM (Protein family), GO (Gene Ontology), KOG (euKaryotic Orthologous Groups), KEGG (Kyoto Encyclopedia of Genes and Genomes).

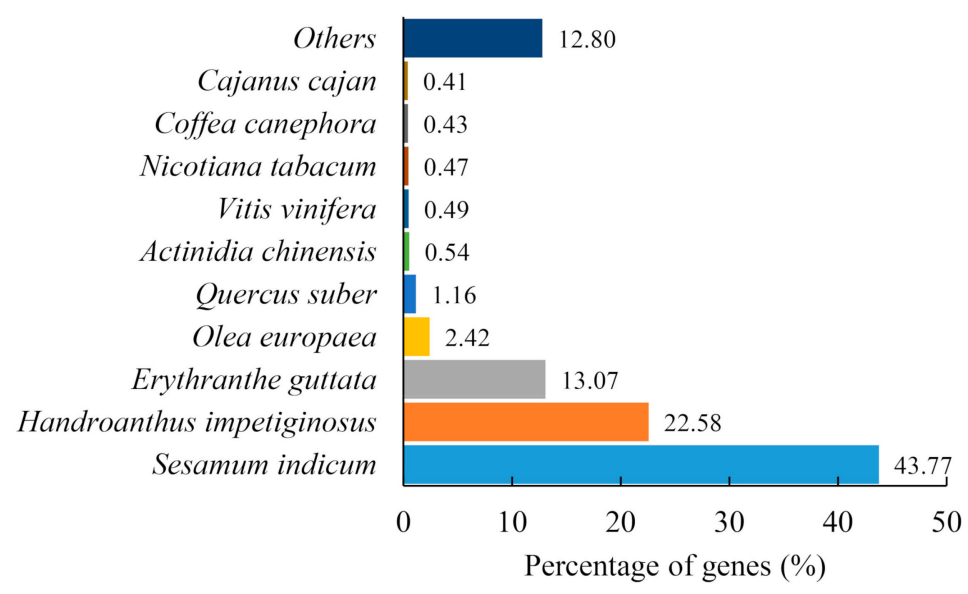

Figure 2. Species distribution of top 10 BLASTx hits against the NR database.

In coding sequence prediction analysis, unigenes were aligned first to the NR and then Swissprot database. If aligned, ORF (open reading frame) information of transcripts was extracted from the alignment results and the sequence of the coding region was translated into amino acid sequences according to the standard codon table. If failed, ESTSCAN (Expression Sequence Tag Scan) software was adopted to predict the ORF of the unigenes. As a result, a total of 157,392 peptides were predicted by BLASTx and the peptide length mainly ranged from 38 to 1059 (Figure 3a) while 35,339 peptides were predicted by ESTSCAN and the peptide length was from 15 to 1092 (Figure 3b). 


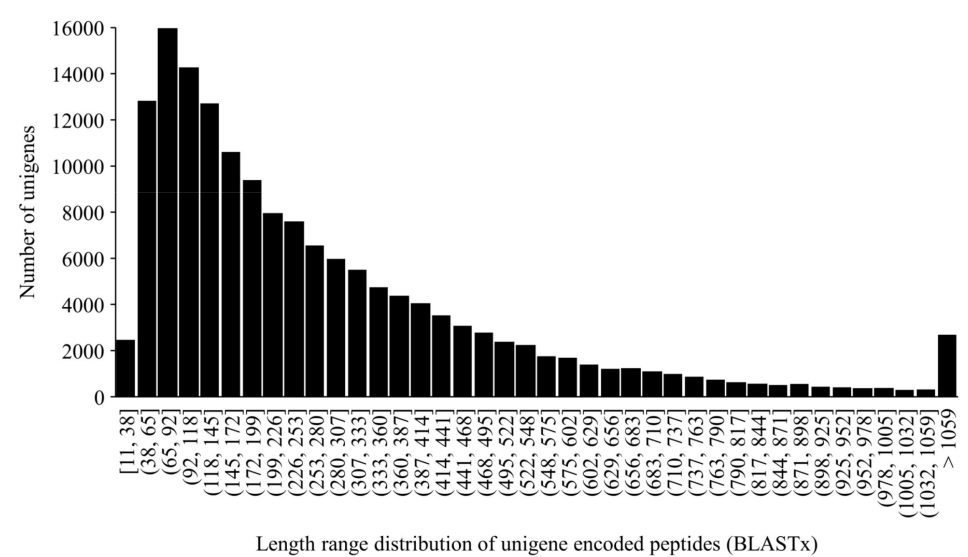

(a)

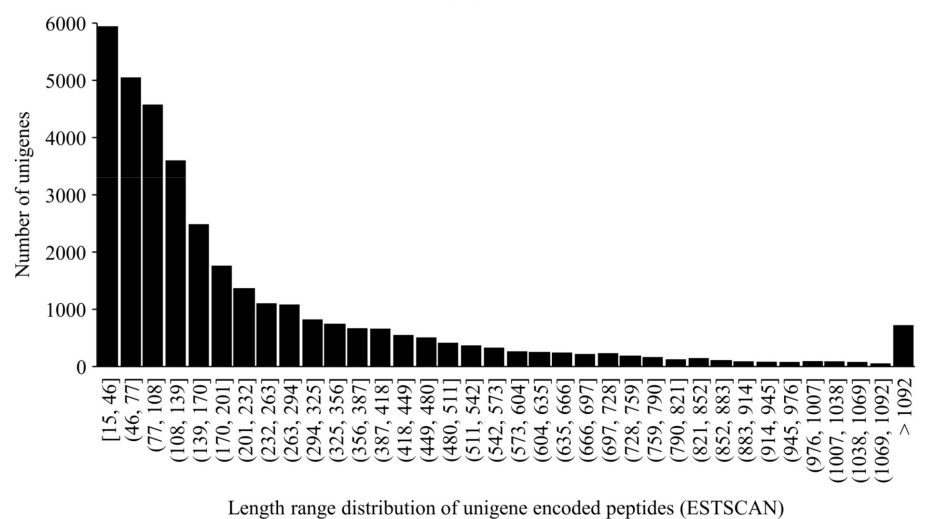

(b)

Figure 3. Length range distributions of unigene encoded peptides: (a) Peptides predicted by BLASTx searches against NR and Swissprot databases; (b) peptides predicted by software ESTSCAN 3.0.3.

To figure out the biological processes that our unigenes are involved in as well as their molecular functions and the cellular environments they reside in, all unigenes were searched against the GO database with software BLAST2GO. Out of 153,198 unigenes, 98,364 (64.20\%) were successfully annotated and classified into three GO categories-biological process (BP), cellular component (CC), and molecular function (MF) - and then assigned to 55 functional groups (Figure 4). As shown in Figure 4 , assignments which fell under BP $(273,598,47.25 \%)$ ranked the highest, followed by CC $(175,650,30.34 \%)$ and MF $(129,742,22.41 \%)$. Similar to R. glutinosa [41] and adventitious roots in Panax ginseng [42], "cellular process" (60,529, 61.54\%) and "metabolic process" (56,468, 57.41\%) were the two most representative subcategories in the BP category, which suggested that lots of important cellular processes and metabolic activities took place in C. grandiflora benth. Unlike adventitious roots in P. ginseng [42], although unigenes related to "cell" $(33,946,34.51 \%)$ and "cell part" $(33,946$, $34.51 \%$ ) were dominant in the CC category, the percentages in C. grandiflora Benth were far less than in $P$. ginseng, which implied that many tissues and organs in C. grandiflora Benth were in construction at a slow speed. In the MF category, the majority of unigenes were involved in "binding" $(59,157$, $60.14 \%)$ and "catalytic activity" $(49,089,49.91 \%)$ in C. grandiflora Benth, and this was somewhat similar to R. glutinosa [41], in which unigenes annotated into "binding" were about $20 \%$ more than "catalytic activity", indicating that more catalytic reactions may occur in the form of protein complexes. 


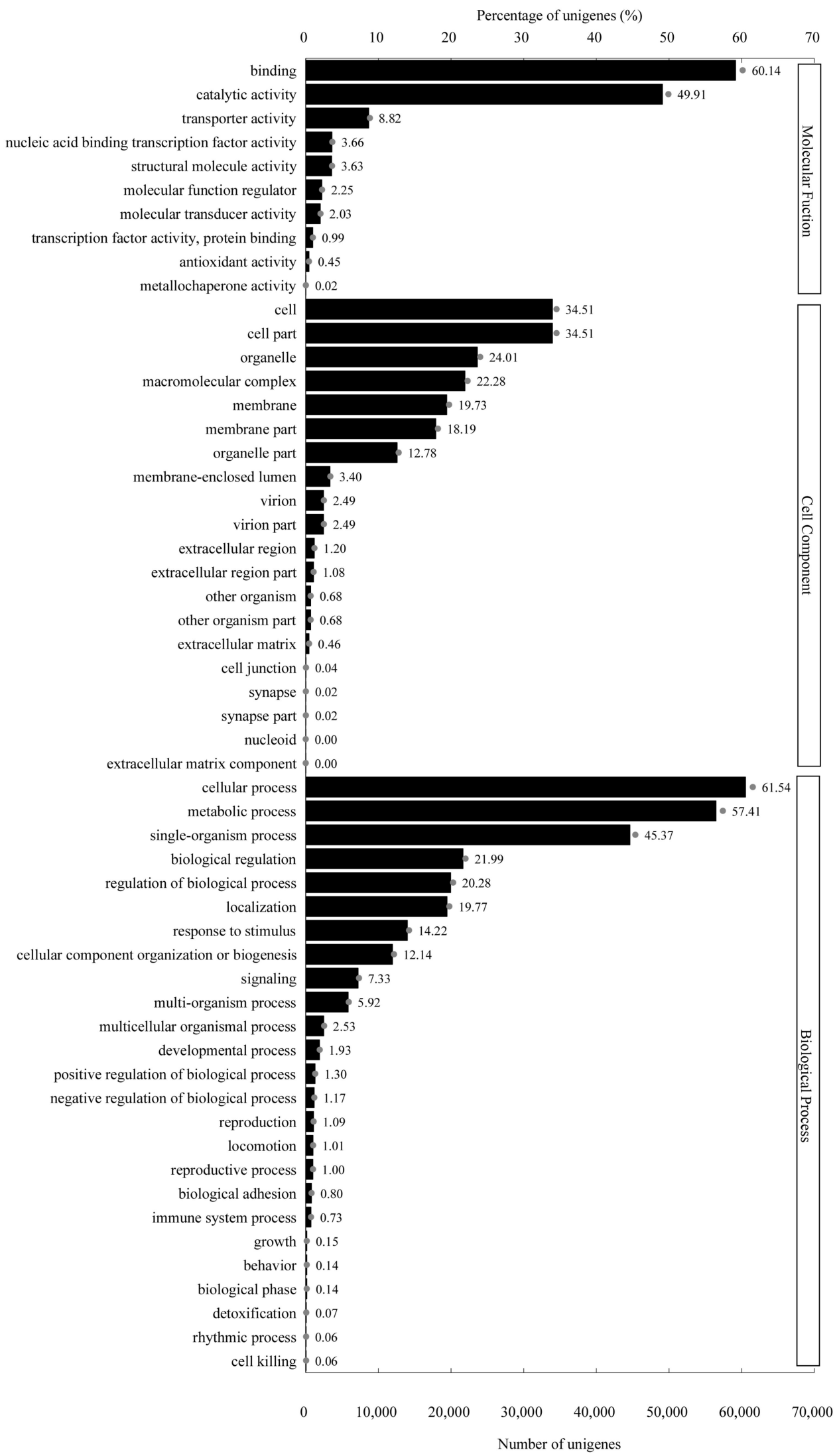

Figure 4. GO classification map: The ordinate represents the next-level GO term of the three GO categories, while the abscissa represents the number of genes annotated into the corresponding term and its proportion of the total number of annotated genes. Three basic categories of GO term, from top to bottom, are the molecular function, cell components, and biological processes. 
KOG refers to clusters of orthologous groups from different eukaryotic species, and genes from the same ortholog are assumed to have the same function. To further classify our unigenes, KOG annotation was performed with software diamond. A total of 44,170 unigenes were classified into 26 KOG groups (Figure 5), where the "posttranslational modification, protein turnover, and chaperon" (5516, 12.49\%) category accounted for the most frequent group, "general function prediction only" $(5445,12.33 \%)$ was the second largest group, and "translation, ribosomal structure, and biogenesis" (4404, 9.97\%) and "intracellular trafficking, secretion, and vesicular transport" (3504, 7.93\%) were tied for the third largest. In addition, 773 unigenes were assigned to "secondary metabolites biosynthesis, transport, and catabolism", implying that catalpol, acteoside, and carotenoid biosynthesis may take place in C. grandiflora Benth.

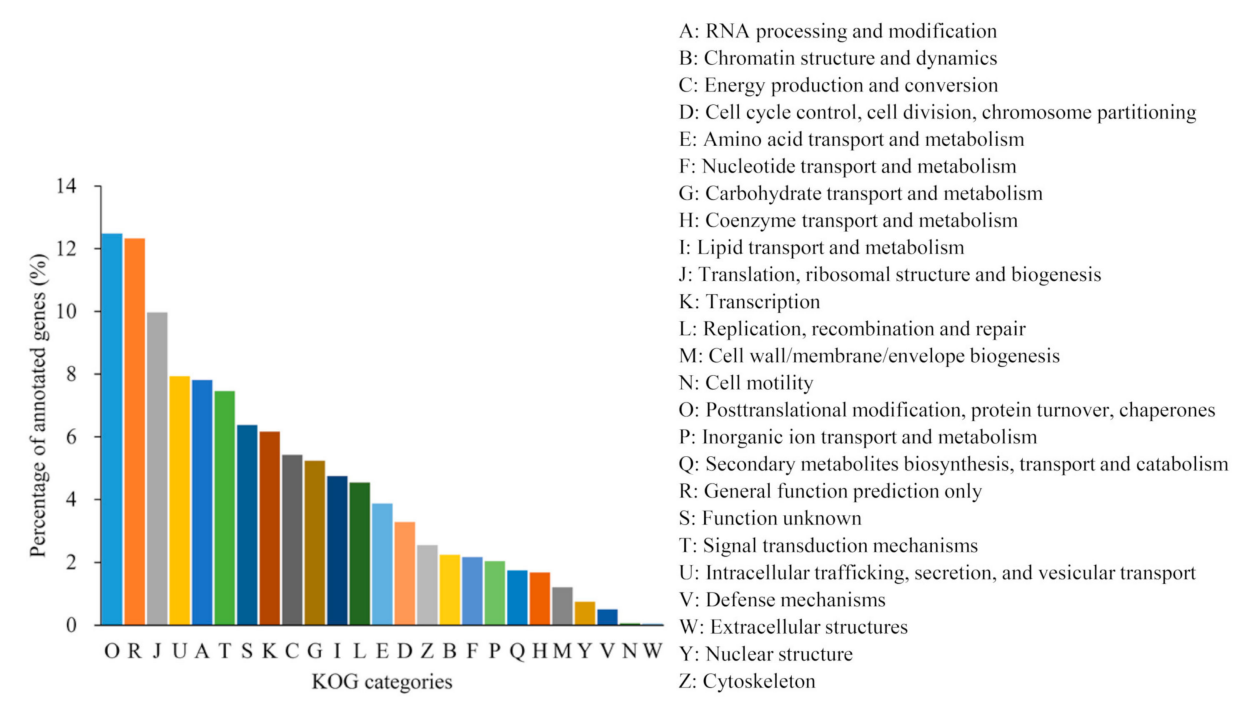

Figure 5. KOG classification map: The abscissa represents KOG groups, while the vertical axis represents the percentage of annotated genes.

KEGG is a database in which gene products and compounds of the cellular metabolic pathways and the functions of these gene products were systematically analyzed. To figure out the active pathways in growing C. grandiflora Benth, KEGG annotation of our unigenes were performed with KAAS (KEGG Automatic Annotation Server). A total of 57,190 (37.33\%) unigenes were annotated into five categories (level 1; cellular processes, environmental information processing, genetic information processing, metabolism, and organismal systems), 19 subcategories (level 2, Figure 6), and 130 pathways (level 3). Similar to R. glutinosa [41], the five pathways with the largest number of genes were "carbohydrate metabolism" (4996, 8.74\%), "overview" (3455, 6.04\%), "amino acid metabolism", "lipid metabolism", and "energy metabolism" in the metabolism category, indicating that primary metabolism was very important to the growth of $C$. grandiflora Benth. In the category of genetic information processing, the two pathways with the largest number of genes were "translation" (5427, 9.49\%) and "folding, sorting, and degradation" (4178, $7.31 \%)$, indicating that protein biosynthesis and processing were more active in C. grandiflora Benth (Figure 6). The numbers of unigenes for "amino acid metabolism", "metabolism of terpenoids and polyketides", and "biosynthesis of other secondary metabolites" were 2927, 1164, and 882 , respectively. These results indicate that the amino acid pathway and terpenoid pathways were active in growing $C$. grandiflora Benth and that the corresponding genes would be good candidate genes for catalpol, acteoside, and carotenoid biosynthesis. 


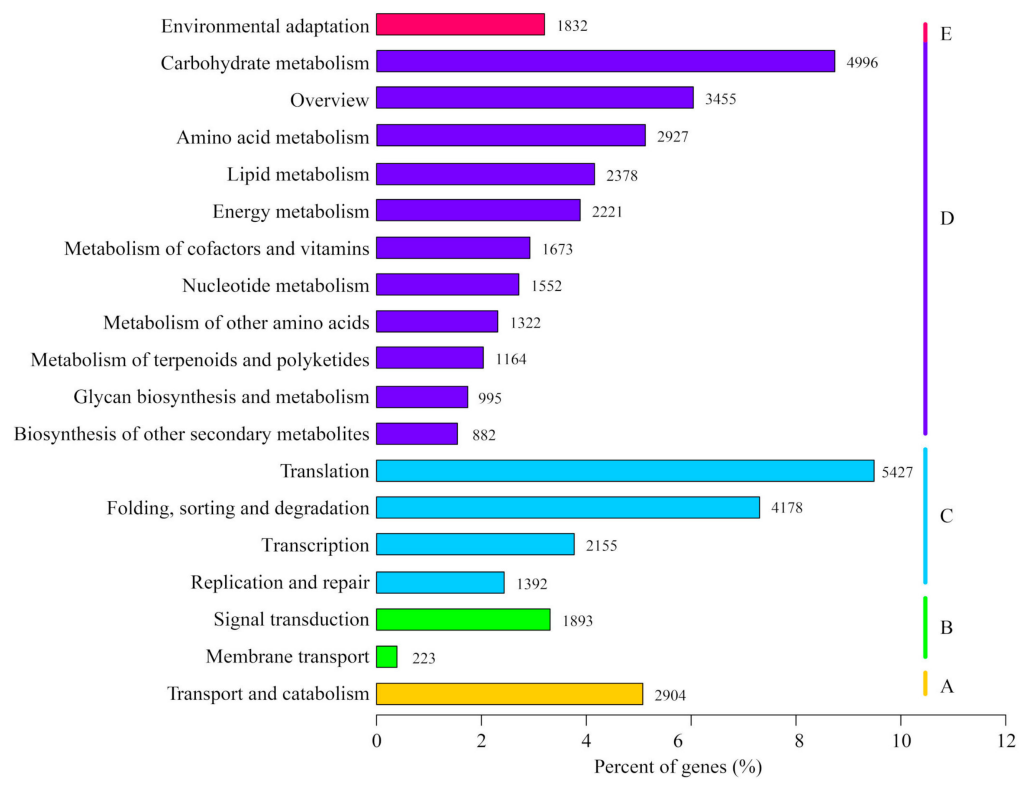

Figure 6. KEGG classification map: The ordinate is the pathway, and the abscissa is the proportion of genes belonging to the corresponding pathway. These genes were divided into five categories: A. Cellular Processes; B. Environmental Information Processing; C. Genetic Information Processing; D. Metabolism; and E. Organismal Systems.

\subsection{Identification of Differentially Expressed Genes (DEGs), GO, and KEGG Enrichment Analysis}

Gene expression level which is transformed from read counts using RSEM (RNA-Seq by Expectation-Maximization) software was analyzed with the FPKM (expected number of Fragments Per Kilobase of transcript sequence per Millions base pairs sequenced) method [43]. According to the criteria $p<0.05$ and $\log _{2}$ (FoldChange) $>1,14,875$ genes ( $9.71 \%$ of all genes) were identified as significant DEGs between leaves and roots, which comprised 8054 upregulated genes $(54.14 \%)$ and 6821 downregulated genes $(45.86 \%$ ) in leaves (Figure $7 \mathrm{a}$, Table S4). There were 4126 genes ( $2.69 \%$ of all genes) identified as significant DEGs between leaves and stems, which comprised 2251 upregulated genes $(54.56 \%)$ and 1875 downregulated genes (45.44\%) in leaves (Figure 7b, Table S5). A total of 9115 genes (5.95\% of all genes) were identified as significant DEGs between stems and roots, which comprised 5290 upregulated genes (58.04\%) and 3825 downregulated genes (41.96\%) in stems (Figure 7c, Table S6). Using a Venn diagram, we compared these three data sets from different comparison groups (C_L vs. C_R, C_S vs. C_R, and C_L vs. C_S). In all three comparison groups, 829 DEGs were identified as being in common (Figure 7d). Specifically, 4839 DEGs were identified in both "C_L vs. C_S" and "C_S vs. C_R" comparisons; 1918 DEGs were identified in both "C_L vs. C_R" and "C_L vs. C_S" comparisons; and 551 DEGs were identified in both "C_L vs. C_S" and "C_S vs. C_R" comparisons (Figure 7d).

GO and KEGG enrichment analysis on all DEGs were performed to find the enriched pathways. The GO enrichment is shown in Tables S7-S9. In the KEGG enrichment analysis, the top two enriched pathways were flavonoid biosynthesis with 37 DEGs and 68 background unigenes, and flavone and flavonol biosynthesis with 10 DEGs and 14 background unigenes in the C_L vs. C_R comparison (Figure 8a, Table S10). In the C_L vs. C_S comparison, the top two pathways were flavone and flavonol biosynthesis with 5 DEGs and 14 background unigenes, and the stilbenoid, diarylheptanoid, and gingerol biosynthesis with 11 DEGs and 48 background unigenes (Figure 8b, Table S11). Finally, in the L_S vs. L_R comparison, the top two pathways were stilbenoid, diarylheptanoid, and gingerol biosynthesis with 15 DEGs and 48 background unigenes, and flavonoid biosynthesis with 3 DEGs and 11 background unigenes (Figure 8c, Table S12). Notably, pathways for both phenylpropanoid biosynthesis and carotenoid biosynthesis were enriched in all three comparisons. The top 20 KEGG enrichment pathways are shown in Figure 8. 


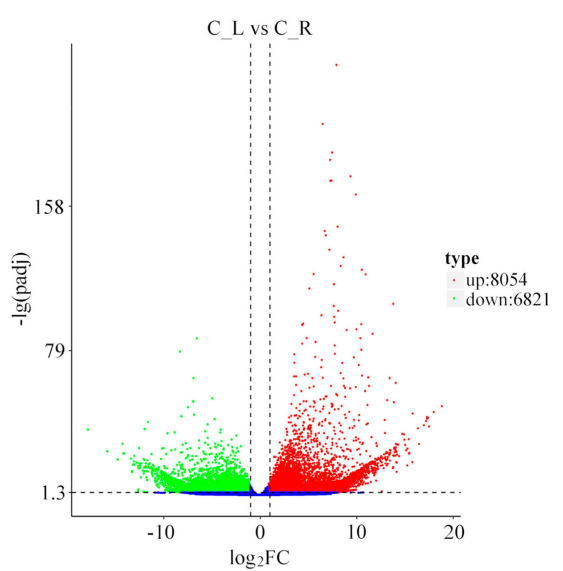

(a)

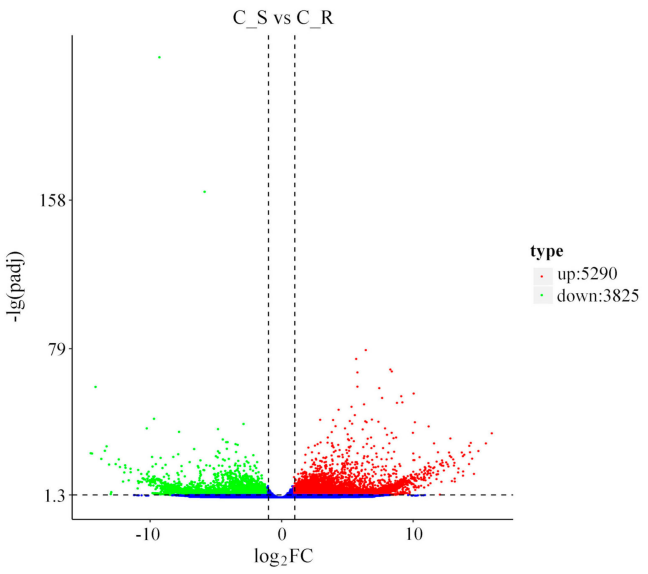

(c)

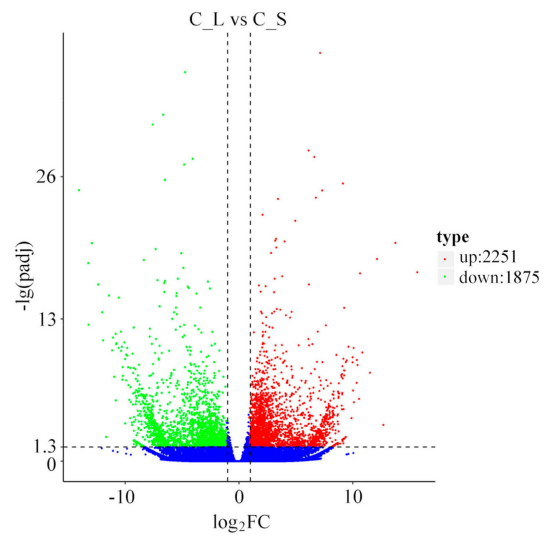

(b)

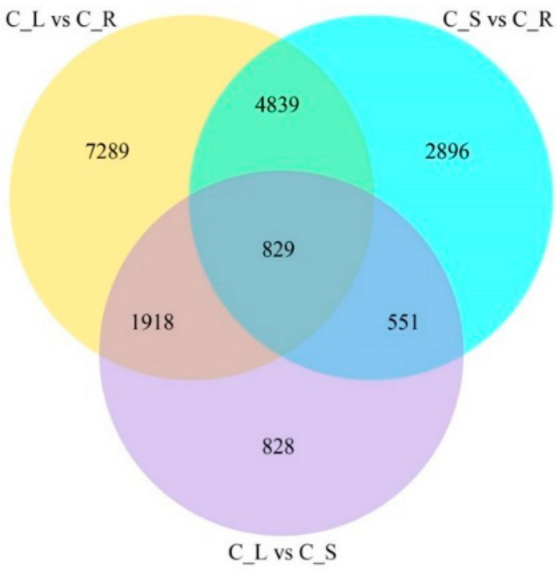

(d)

Figure 7. Differentially expressed genes (DEGs) in different comparisons. Volcano plots of the DEGs in different comparisons: The red dots mean significantly upregulated genes, and the green dots represent significantly downregulated genes. The black dots represent non-DEGs. (a) C_L vs. C_R volcano; (b) C_L vs. C_S volcano; and (c) C_S vs. C_R volcano. (d) Venn diagram of DEGs in different comparisons: All DEGs are clustered into three comparison groups represented by three circles. Overlapping parts of the different circles represent the number of DEGs in common among those comparison groups.

\subsection{Biosynthetic Genes of the Terpenoid Backbone and Catalpol in C. grandiflora Benth}

Terpenoids are produced from the universal precursor IPP (a five-carbon building material) and its isomer DMAPP [44]. In plants, IPP is synthesized via the cytoplasmic MVA pathway from acetyl-CoA and through the plastidial MEP pathway from glyceraldehyde 3-phosphate and pyruvate; IPP isomerase (IDI) catalyzes the interconversion between IPP and DMAPP [44] (Figure 9a). When examining the annotation of unigenes against the KEGG database, 239 unigenes were assigned to the terpenoid backbone biosynthesis pathway, including 74 unigenes encoding 6 enzymes in the MVA pathway and 165 unigenes encoding 8 enzymes in the MEP pathway (Table 4). Among these genes, the largest number is DXS (67), followed by IDI (28), AACT (24), and CMK (24), and the lowest number is MCS (1). Transcriptome profiling data showed that the MEP pathway is more active in leaves, while the MVA pathway is more active in stems due to the high expression levels of corresponding pathway genes (Figure 9b). 


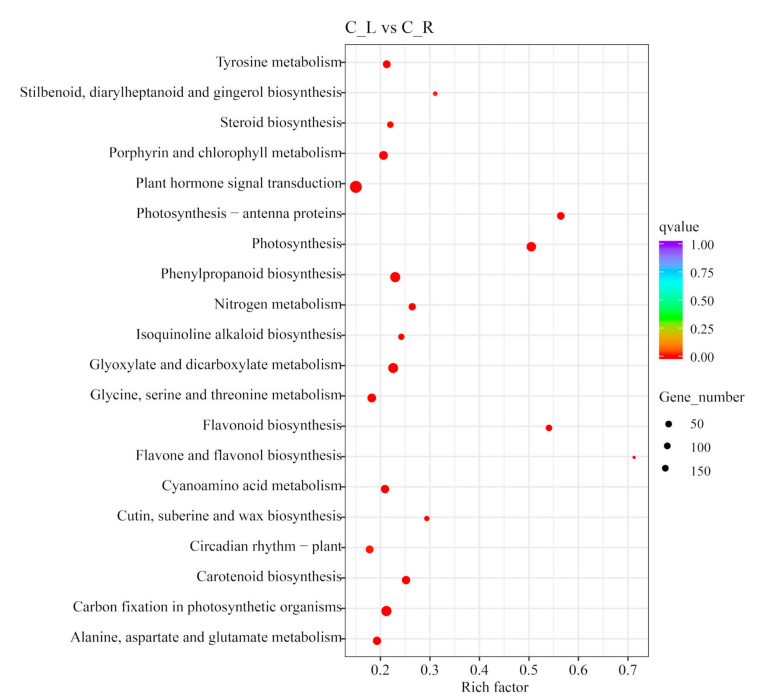

(a)

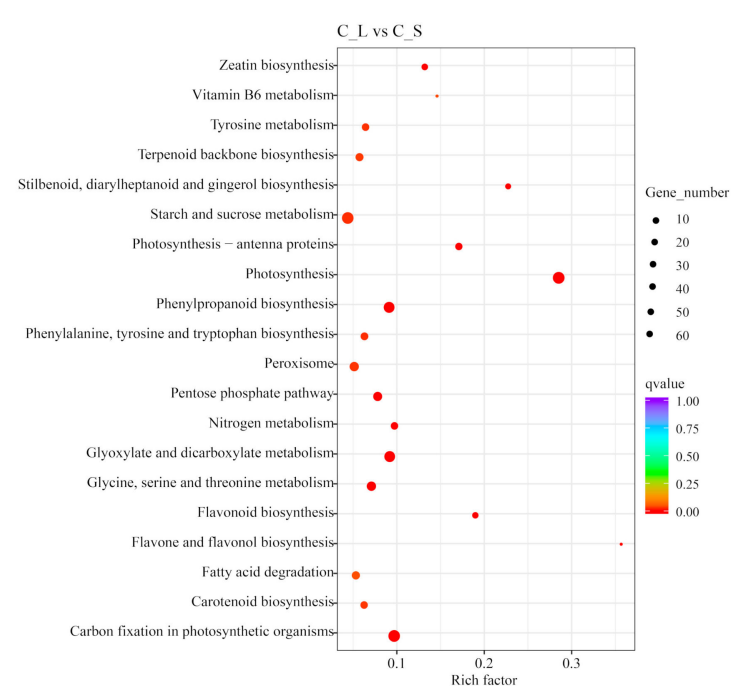

(b)

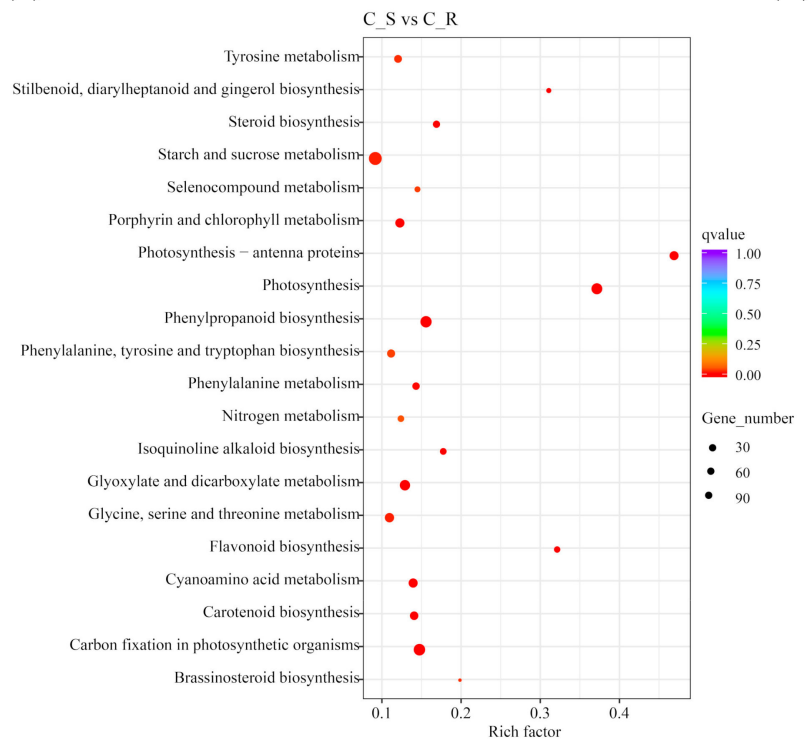

(c)

Figure 8. Top 20 of KEGG pathway enrichment of DEGs: The y-axis indicates the pathway name, and the $x$-axis indicates the enrichment factor corresponding to the pathway. The q-value is represented by the color of the dot. The number of DEGs is represented by the size of the dots. (a) C_L vs. C_R; (b) C_L vs. C_S; and (c) C_S vs. C_R.

Catalpol, belonging to iridoid glucoside, is usually found in Scrophulariaceae plants $[6,45,46]$, and iridoid glucosides are derived from MEP and MVA pathways [41]. Based on feeding experiments with isotope labeling and transcriptome analysis, the draft biosynthesis pathway of catalpol was first proposed in R. glutinosa in $2012[47,48]$. Then, the complete pathway of catalpol was clarified for the first time in P. kurroa in 2015 [17].

According to the KEGG and Swissprot annotation, a total of 368 unigenes were assigned to the catalpol biosynthetic pathway, with 60 unigenes upregulated and 39 unigenes downregulated in leaf vs. root (Figure 9a and Table 4). The unigenes encoding 13 enzymes involved in catalpol biosynthesis are listed in Table 4. Among these genes, the largest number was $A L D H$ (76), followed by $8 H G O$ (53), IO (44), GPPS (32), and UGT (30), and the lowest number was F3D (2) (Table 4). Like the terpenoid backbone pathway, most genes in the catalpol biosynthesis pathway possess at least two unigenes, displaying the redundancy of the plant genes and adding the difficulty of deciphering the pathway 
(Table 4). In our transcriptome, unigenes of catalpol pathways are more abundant in leaves, as revealed by much higher expression level of GES, G10H, IS, IO, and F3D in leaves than in roots (Figure 9b, Table 4). It is worth noting that there were only two F3D genes in our transcriptome and that it was only expressed in leaves and stems, but not roots, which indicated that the catalpol biosynthesis was active in aboveground growth at this developmental stage (Figure 9a, Table 4). Therefore, while it is the roots of $C$. grandiflora Benth that are used as medicinal materials, our results imply that the catalpol is first synthesized in the leaves and then transported and stored in the roots. Furthermore, $D C H$ gene functioning in the conversion of deoxygeniposidic acid to geniposidic acid was not found in our transcriptome, which may be a result of low expression or low homology with the known $D C H$ genes.

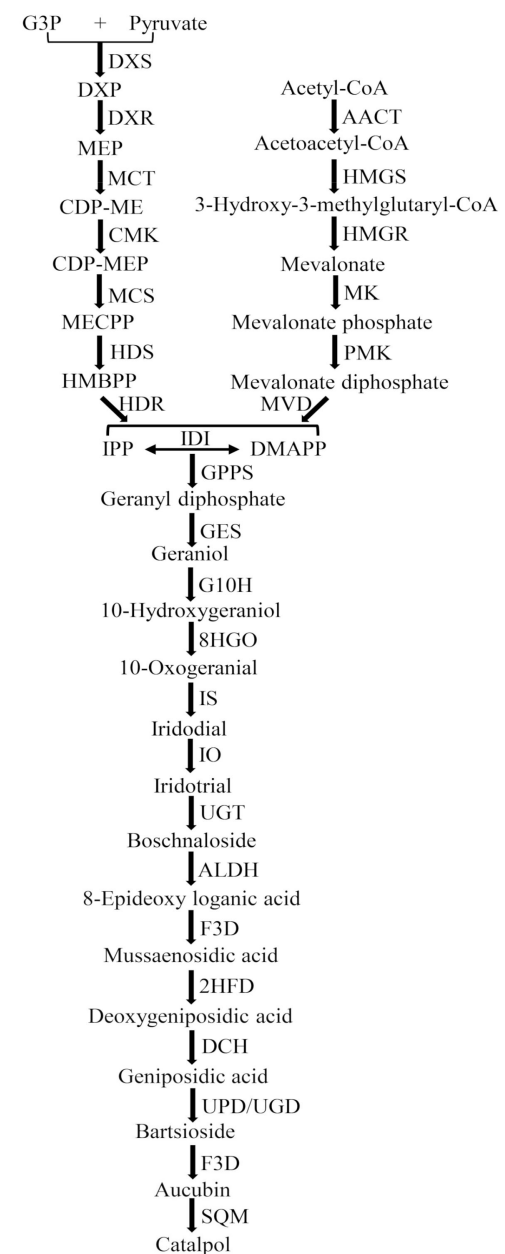

(a)

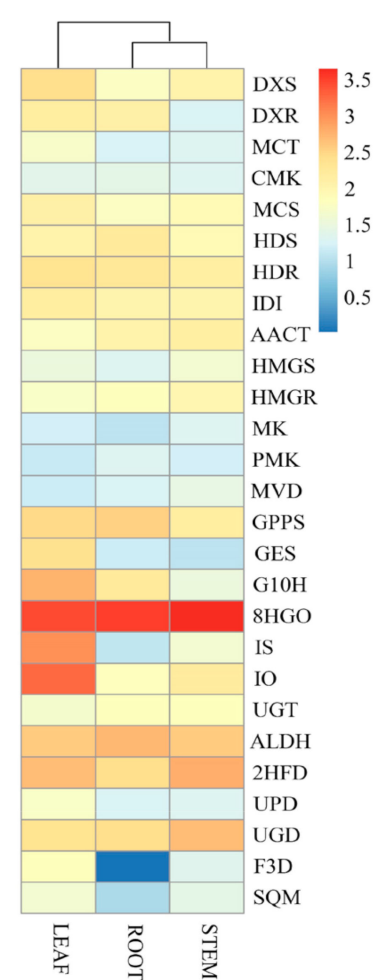

(b)

Figure 9. Expression of unigenes in the putative pathway of terpenoid backbone and catalpol biosynthesis in C. grandiflora Benth: (a) Proposed biosynthetic pathway of terpenoid backbone and catalpol. (b) Heatmap based on the expression level of unigenes involved in terpenoid backbone and catalpol biosynthesis across three tissues in C. grandiflora Benth. The expression level is the sum of all the unigenes for each gene, and $\log _{10}(\operatorname{sum}(\mathrm{FPKM})+1)$ was used to plot the heatmap. Candidate unigenes were selected according to the annotation. Abbreviations: G3P, Glyceraldehyde 3-phosphate; DXP, 1-deoxy-D-xylulose-5-phosphate; MEP, 2-C-methyl-Derythritol 4-phosphate; CDP-ME, 4-(Cytidine 5'-diphospho)-2-C-methyl-D-erythritol; MECPP, 2-C-methyl-D-erythritol-2,4-cyclodiphosphate; HMBPP, 1-hydroxy-2-methyl-2-butenyl 4-diphosphate; IPP, isopentenyl pyrophosphate; DMAPP, dimethylallyl pyrophosphate. 
Table 4. Putative genes of the mevalonate (MVA), 2-C-methyl-D-erythritol-4-phosphate (MEP), and catalpol biosynthesis pathways.

\begin{tabular}{|c|c|c|c|c|c|c|}
\hline Pathway & Gene & Gene Name & EC & Number & $\begin{array}{c}\text { Upregulated } \\
\left(\log _{2}(\mathrm{FC})>1,\right. \\
\text { L vs. R) }\end{array}$ & $\begin{array}{c}\text { Downregulated } \\
\left(\log _{2}(\mathrm{FC})>1, \mathrm{~L}\right. \\
\text { vs. R) }\end{array}$ \\
\hline \multirow{6}{*}{ MVA } & $A A C T$ & acetyl-CoA C-acetyltransferase & 2.3.1.9 & 24 & \multirow{3}{*}{2} & \multirow[t]{4}{*}{2} \\
\hline & HMGS & hydroxymethylglutaryl-CoA synthase & 2.3.3.10 & 8 & & \\
\hline & $H M G R$ & hydroxymethylglutaryl-CoA reductase & 1.1.1.34 & 9 & & \\
\hline & $M K$ & mevalonate kinase & 2.7.1.36 & 6 & \multirow[t]{3}{*}{1} & \\
\hline & $P M K$ & phosphomevalonate kinase & 2.7.4.2 & 21 & & 3 \\
\hline & $M V D$ & diphosphomevalonate decarboxylase & 4.1.1.33 & 6 & & \\
\hline \multirow{8}{*}{ MEP } & $D X S$ & 1-deoxy-D-xylulose-5-phosphate synthase & 2.2.1.7 & 67 & \multirow[t]{2}{*}{12} & \\
\hline & DXR & $\begin{array}{l}\text { 1-deoxy-D-xylulose-5-phosphate } \\
\text { reductoisomerase }\end{array}$ & 1.1.1.267 & 10 & & \\
\hline & $M C T$ & $\begin{array}{c}\text { 2-C-methyl-D-erythritol 4-phosphate } \\
\text { cytidylyltransferase }\end{array}$ & 2.7.7.60 & 3 & \multirow[t]{2}{*}{1} & \\
\hline & CMK & $\begin{array}{l}\text { 4-diphosphocytidyl-2-C-methyl-D-erythritol } \\
\text { kinase }\end{array}$ & 2.7.1.148 & 24 & & \\
\hline & MCS & $\begin{array}{l}\text { 2-C-methyl-D-erythritol 2,4-cyclodiphosphate } \\
\text { synthase }\end{array}$ & 4.6.1.12 & 1 & \multirow[t]{3}{*}{1} & \\
\hline & HDS & $\begin{array}{l}\text { (E)-4-hydroxy-3-methylbut-2-enyl-diphosphate } \\
\text { synthase }\end{array}$ & 1.17.7.1/1.17.7.3 & 21 & & \\
\hline & $H D R$ & $\begin{array}{l}\text { 4-hydroxy-3-methylbut-2-en-1-yl diphosphate } \\
\text { reductase }\end{array}$ & 1.17.7.4 & 11 & & \\
\hline & $I D I$ & isopentenyl pyrophosphate isomerase & 5.3.3.2 & 28 & 2 & \\
\hline \multirow{13}{*}{ Catalpol } & GPPS & geranyl diphosphate synthase & 2.5.1.1 & 32 & 5 & \multirow[t]{2}{*}{6} \\
\hline & GES & geraniol synthase & 3.1.7.11 & 9 & 3 & \\
\hline & $\mathrm{G} 10 \mathrm{H}$ & geraniol 10-hydroxylase & 1.14.13.152 & 14 & 8 & 2 \\
\hline & $8 H G O$ & 8-hydroxygeraniol oxidoreductase & 1.1.1.324 & 53 & 13 & 11 \\
\hline & IS & iridoid synthase & \multirow[t]{2}{*}{ 1.3.1.99 } & 5 & 3 & \\
\hline & $I O$ & iridoid oxidase & & 44 & 3 & 2 \\
\hline & UGT & UDP-glucosyl transferase & 2.4 .1 & 22 & 4 & 4 \\
\hline & $A L D H$ & aldehyde dehydrogenase & 1.2.1.3 & 76 & 7 & 10 \\
\hline & $F 3 D$ & flavanone 3-dioxygenase & 1.14.11.9 & 2 & 1 & \\
\hline & $2 H F D$ & 2-hydroxyisoflavanone dehydratase & 4.2.1.105 & 10 & 2 & \\
\hline & $U P D$ & uroporphyrinogen decarboxylase & 4.1.1.37 & 23 & 3 & \\
\hline & $U G D$ & UDP-glucuronic acid decarboxylase & 4.1.1.35 & 70 & 3 & 4 \\
\hline & $S Q M$ & squalene monooxygenase & 1.14.13.132 & 8 & 5 & \\
\hline
\end{tabular}

Note: FC represents fold change.

\subsection{Biosynthetic Genes of Acteoside in C. grandiflora Benth}

Studies have shown that acteoside is widely distributed in more than 150 plant species and has medicinal properties including antioxidant, anti-inflammation, anti-nephritis, cell regulation, hepatoprotection, immunoregulation, and neuroprotection [49]. Upstream regions of the acteoside biosynthetic pathway, including the phenylalanine-derived pathway and tyrosine-derived pathway, was first clarified in Olea europaea using feeding experiments, while the downstream is largely unknown [26]. The downstream region was partially deciphered with elicitor inducing and transcriptome sequencing in R. glutinosa [18,28].

Based on the KEGG annotation in this study, a total of 213 unigenes were assigned to the acteoside biosynthetic pathway, with 40 unigenes significantly upregulated and 16 unigenes significantly downregulated in leaves vs. roots (Figure 10a). The unigenes encoding key enzymes involved in acteoside biosynthesis are listed in Table 5. Among these genes, the largest number was CuAO (53), followed by 4CL (35), ADH (26), and UGT (22), and the lowest number was HCT (6) (Table 5). In the DEGs analysis, four genes including $P A L, C 4 H, C 3 H$, and $4 C L$ were upregulated in leaves and stems compared with roots (Figure 10b, Table 5), which implies that the phenylalanine-derived pathway is active in aerial parts. 


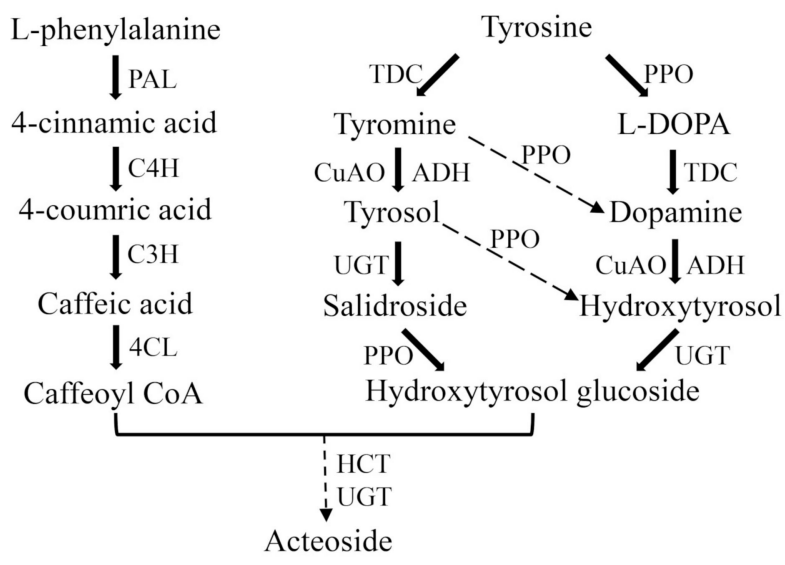

(a)

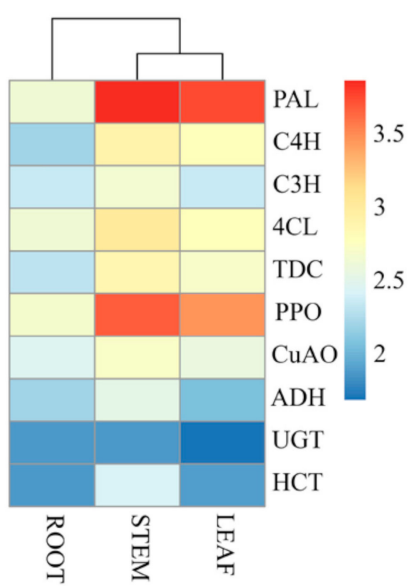

(b)

Figure 10. Expression of unigenes in putative pathways of acteoside biosynthesis in C. grandiflora Benth: (a) Proposed biosynthetic pathway of acteoside. The solid arrow represents the known steps, and the dashed arrows denote the putative steps. (b) Heatmap based on the expression level of unigenes involved in acteoside biosynthesis across three tissues: Candidate unigenes were selected according to the annotation.

Table 5. Putative genes of acteoside biosynthesis pathways.

\begin{tabular}{|c|c|c|c|c|c|}
\hline Gene & Gene name & EC & Number & $\begin{array}{c}\text { Upregulated } \\
\left(\log _{2}(\mathrm{FC})>1,\right. \\
\text { L vs. R) }\end{array}$ & $\begin{array}{c}\text { Downregulated } \\
\left(\log _{2} \text { (FC) }>1\right. \\
\text { L vs. R) }\end{array}$ \\
\hline$P A L$ & phenylalanine ammonia-lyase & 4.3.1.24 & 19 & 4 & 1 \\
\hline $\mathrm{C} 4 \mathrm{H}$ & cinnamate-4-hydroxylase & 1.14.14.91 & 12 & 5 & \\
\hline $\mathrm{C} 3 \mathrm{H}$ & coumarate-3-hydroxylase & 1.14.14.96 & 10 & 3 & 1 \\
\hline $4 C L$ & 4-coumarate-CoA ligase & 6.2.1.12 & 35 & 9 & \\
\hline$T D C$ & tyrosine decarboxylase & 4.1.1.25 & 19 & 3 & \\
\hline $\mathrm{CUAO}$ & copper-containing amine oxidase & 1.4.3.21 & 53 & 4 & 2 \\
\hline$A D H$ & alcohol dehydrogenase & 1.1.1.1 & 26 & 3 & 3 \\
\hline UGT & UDP-glucose glucosyltransferase & 2.4.1.35 & 22 & 4 & 4 \\
\hline$P P O$ & polyphenol oxidase & 1.14 .18 .1 & 11 & 4 & 5 \\
\hline HCT & $\begin{array}{c}\text { Shikimate } \\
\text { O-hydroxycinnamoyltransferase }\end{array}$ & 2.3.1.133 & 6 & 1 & \\
\hline
\end{tabular}

\subsection{Biosynthetic Genes of Azafrin in C. grandiflora Benth}

Recent studies have shown that azafrin can significantly improve myocardial contractile function during myocardial ischemia via activation of the Nrf2-ARE (Nuclear factor-erythroid 2-related factor-Antioxidant Response Element) pathway in rats [7,33]. So far, biosynthesis and chemical synthesis pathways of azafrin are still unknown. Azafrin is a derivative of carotenoid, a tetraterpenoid compound [38]. In higher plants, carotenoids are manufactured in plastid with IPP generated by the MEP pathway [50]. The putative carotenoid biosynthesis pathway, including the MEP part, lutein branch, strigolactone branch, capsanthin/capsorubin branch, abscisic acid branch, and without the azafrin pathway, has been established in plants (Figure 11a) $[35,51]$. However, studies have shown that the substrate $\beta$-carotene can be directly converted into $10^{\prime}$-apo- $\beta$-carotenal and ionone by $\beta$-carotene- $9^{\prime}, 10^{\prime}$-oxygenase (BCO2) in non-plants or can be indirectly converted into $10^{\prime}$-apo- $\beta$-carotenal and ionone by DWARF27 and carotenoid cleavage dioxygenases 7 (CCD7) in plants [39,52]. The differences between azafrin and $10^{\prime}$-apo- $\beta$-carotenal are one terminal carboxyl group and two hydroxyl groups in the cyclohexane skeleton. From the aspect of biochemistry, acetaldehyde dehydrogenase (ALDH) can transform aldehyde into carboxylic acid and the cytochrome P450 monooxygenases (CYP450s) are capable of inserting oxygen atoms into inert hydrophobic molecules to make them more hydrophilic [53]. There is also a report that post-modification of 
terpenoid derivatives is mostly initiated by oxidation and that most of them are catalyzed by CYP450s and then other post-modification reactions are carried out on the basis of oxidation products [54]. Then, we hypothesize that azafrin is produced in two continuous steps: $10^{\prime}$-apo- $\beta$-carotenal is first oxidized by ALDH and then is hydrolyzed by CYP450. The detailed biosynthetic pathway of azafrin is shown in Figure 11a.

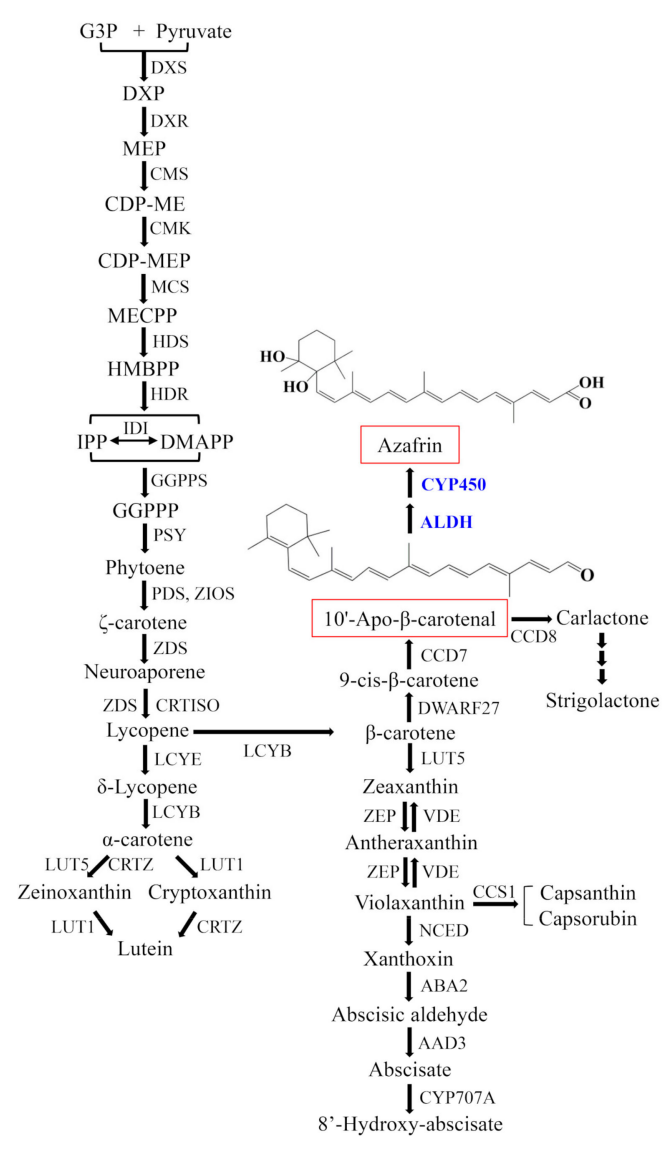

(a)

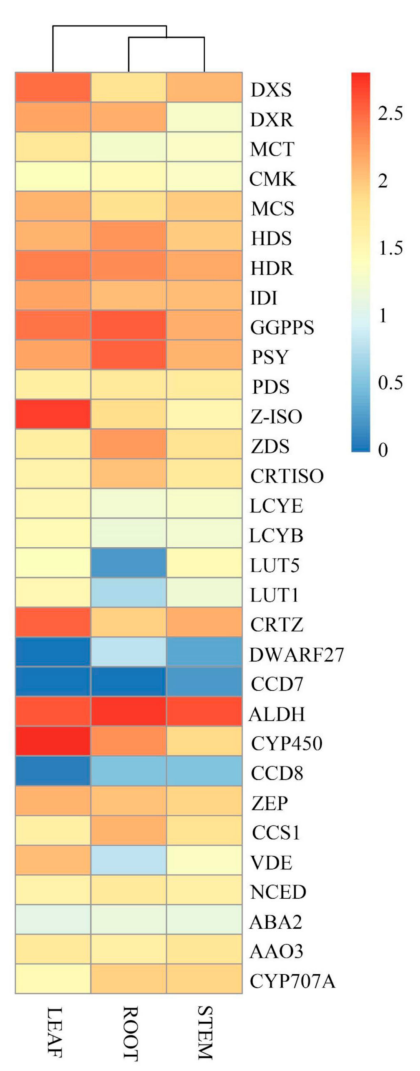

(b)

Figure 11. The putative carotenoid biosynthesis pathway and the heatmap of corresponding genes in C. grandiflora Benth: (a) Proposed biosynthetic pathway of carotenoid. Here, the hypothesis that $10^{\prime}$-apo- $\beta$-carotenal can be converted into azafrin by ALDH and CYP450 is proposed. (b) Heatmap based on the expression level of unigenes involved in carotenoid biosynthesis across three tissues. For CYP450, only genes of $\log _{2}$ (FC) > 10 (leaf vs. root) were selected for map. Candidate unigenes were selected according to the annotation.

Based on the KEGG annotation and NR annotation in this study, a total of 356 unigenes were correlated with the carotenoid biosynthesis, of which 161 unigenes were assigned to the azafrin biosynthetic pathway with 20 unigenes upregulated and 33 unigenes downregulated in leaves vs. roots (Table 6). For the MEP portion, it was active in leaves, stems, and roots in general as it is known to provide the universal precursor for the terpenoids (Figure 11b). For the lutein pathway, it was more active in leaves and stems while somewhat inactive in roots due to the low expressions of LUT5 and LUT1 genes (Figure 11b). For the azafrin and strigolactone branch, it was slightly active in stem, as neither DWARF27 and CCD7 were expressed in leaves nor CCD7 were expressed in roots (Figure 11b). For the capsanthin/capsorubin and abscisic acid branch, it is more active in leaves and stems and somewhat blocked by the low expression of the LUT5 gene (Figure 11b). What should be noted here is that there is only one gene encoding the CCD7 enzyme, which may be a rate-limiting enzyme (Table 6). 
Table 6. Putative genes of carotenoid biosynthesis pathways.

\begin{tabular}{|c|c|c|c|c|c|}
\hline Gene & Gene Name & EC & Number & $\begin{array}{c}\text { Upregulated } \\
\left(\log _{2}(\text { FC) }>1,\right. \\
\text { L vs. R) }\end{array}$ & $\begin{array}{c}\text { Downregulated } \\
\left(\log _{2} \text { (FC) }>1\right. \\
\text { L vs. R) }\end{array}$ \\
\hline GGPPS & geranylgeranyl diphosphate synthase & 2.5.1.29 & 15 & 3 & 6 \\
\hline PSY & phytoene synthase & 2.5.1.32 & 9 & 1 & 2 \\
\hline PDS & phytoene desaturase & 1.3.5.5 & 7 & 2 & 1 \\
\hline Z-ISO & zeta-carotene isomerase & 5.2.1.12 & 3 & & 2 \\
\hline ZDS & zeta-carotene desaturase & 1.3.5.6 & 26 & 1 & 4 \\
\hline crtISO & carotenoid isomerase & 5.2.1.13 & 10 & & 2 \\
\hline LCYE & lycopene epsilon-cyclase & 5.5.1.18 & 49 & 3 & 1 \\
\hline$L C Y B$ & lycopene beta-cyclase & 5.5.1.19 & 2 & 1 & \\
\hline LUT5 & beta-ring hydroxylase & 1.14.-.- & 18 & 8 & \\
\hline LUT1 & carotenoid epsilon hydroxylase & 1.14.99.45 & 14 & 2 & \\
\hline CRTZ & beta-carotene 3-hydroxylase & 1.14.13.129 & 11 & 6 & 5 \\
\hline DWARF27 & beta-carotene isomerase & 5.2.1.14 & 2 & & 1 \\
\hline CCD7 & $\begin{array}{l}\text { 9-cis-beta-carotene } 9^{\prime}, 10^{\prime} \text {-cleaving } \\
\text { dioxygenase }\end{array}$ & 1.13.11.68 & 1 & & \\
\hline$A L D H$ & aldehyde dehydrogenase & 1.2.1.3 & 76 & 7 & 10 \\
\hline CYP450 & cytochrome P450 & & 10 & 5 & 5 \\
\hline CCD8 & carlactone synthase & 1.13.11.69 & 11 & & \\
\hline ZEP & zeaxanthin epoxidase & 1.14.15.21 & 17 & 1 & 4 \\
\hline CCS1 & capsanthin/capsorubin synthase & 5.3.99.8 & 4 & & 1 \\
\hline$V D E$ & violaxanthin de-epoxidase & 1.23.5.1 & 12 & 3 & \\
\hline NCED & 9-cis-epoxycarotenoid dioxygenase & 1.13.11.51 & 33 & 6 & 7 \\
\hline$A B A 2$ & xanthoxin dehydrogenase & 1.1.1.288 & 5 & 1 & \\
\hline AAD3 & abscisic-aldehyde oxidase & 1.2.3.14 & 11 & & \\
\hline CYP707A & $(+)$-abscisic acid $8^{\prime}$-hydroxylase & 1.14.14.137 & 10 & 4 & 6 \\
\hline
\end{tabular}

\subsection{Identification of Transcription Factors (TFs)}

Transcription factors can activate or inhibit the expression of functional genes in the biosynthetic pathway of plant metabolites, thereby effectively regulating the synthesis and accumulation of secondary metabolites. According to gene sequence alignment to the PFAM database, referring to the Hidden Markov Model files of various TFs, the HMMER3.0 software was used to search the transcriptome database of $C$. grandiflora Benth. The results showed that, in our transcriptome, 4888 unigenes were annotated as TFs belonging to 78 categories. The top three TFs with the largest numbers were MYB (avian myeloblastosis viral oncogene homolog, 356, accounting for 7.28\%), WRKY (WRKY domain-containing protein, 301, accounting for 6.16\%), and orphans (234, accounting for $4.79 \%$ ), followed by $\mathrm{HB}$ (homeobox, 223, accounting for 4.56\%), $\mathrm{C} 3 \mathrm{H}$ (Cys3His zinc finger domain-containing protein, 209, accounting for 4.28\%), and bHLH (basic Helix-Loop-Helix, 201, accounting for 4.11\%) (Figure 12, Table 7, and Table S13). There were also TFs ERF and bZIP (basic region-leucine zipper, Table 7). Among these TFs, most were expressed in both root and leaf tissues, with 121 and 132 showing significantly upregulation and downregulation in leaves, respectively (Table 7).

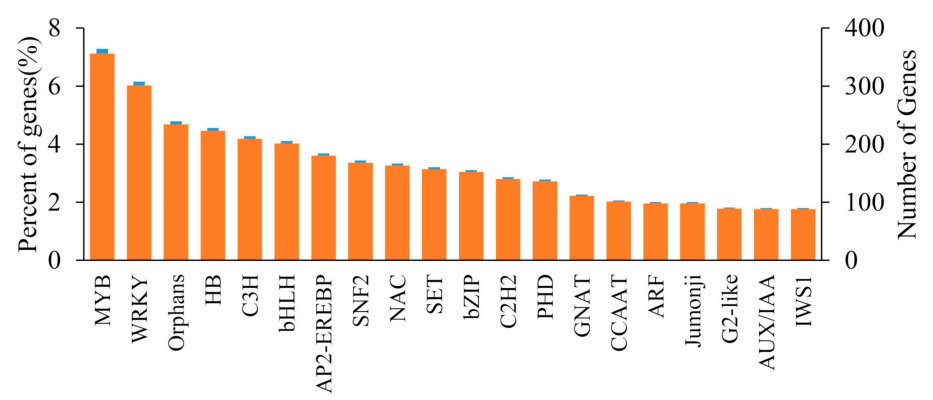

Figure 12. Top 20 transcription factors in C. grandiflora Benth. 
Table 7. Summary of transcription factor unigenes of C. grandiflora Benth.

\begin{tabular}{cccc}
\hline TF Family & $\begin{array}{c}\text { Number of Genes } \\
\text { Detected }\end{array}$ & $\begin{array}{c}\text { UpRegulated in Leaves } \\
\left(\log _{\mathbf{2}}(\mathrm{FC})>\mathbf{2}\right)\end{array}$ & $\begin{array}{c}\text { Upregulated in Roots } \\
\left(\log _{\mathbf{2}}(\mathrm{FC})>\mathbf{2}\right)\end{array}$ \\
\hline MYB & 356 & 25 & 21 \\
WRKY & 301 & 16 & 44 \\
Orphans & 234 & 14 & 11 \\
HB & 223 & 25 & 4 \\
C3H & 209 & 6 & 7 \\
bHLH & 201 & 21 & 9 \\
ERF & 180 & 9 & 21 \\
bZIP & 152 & 5 & 15 \\
Total & 1856 & 121 & 132 \\
\hline
\end{tabular}

Note: TF (Transcription Factor), MYB (avian myeloblastosis viral oncogene homolog), WRKY (WRKY domain-containing protein), $\mathrm{HB}$ (homeobox), $\mathrm{C} 3 \mathrm{H}$ (Cys3His zinc finger domain-containg protein), bHLH (basic Helix-Loop-Helix), ERF(Ethylene Responsive Factor), bZIP (basic region-leucine zipper).

Studies have also shown that the active components of medicinal plants are regulated by many TFs and that the number of genes regulated by a specific TF varies widely. There may be even crosstalk between regulations. In Artemisia annua, only AaHD1 (Homeodomain-leucine zipper) and AaGSW1 (Glandular trichome-Specific WRKY 1) can activate transcription of the CYP71AV1 gene [55,56]; AaWRKY1, AabHLH1, and ERF (Ethylene Response Factor) TFs including AaTAR1 (Trichome and Artemisinin Regulator 1), AaERF1, AaERF2, and AaORA (Octadecanoid-derivative Responsive Apetala2 domain) can activate the transcription of both the amorpha-4,11-diene synthase (ADS) gene and the CYP71AV1 gene and then facilitates artemisinin biosynthesis [57-61]; AabZIP1 is responsible for the activation of the ADS, CYP71AV1, and AaGSW1 genes [62], while AaMYC2 is responsible for the CYP71AV1,DBR2 (Double-Bond Reductase 2), and AaGSW1 genes [63,64]; and AaMYB2 may regulate the ADS, CYP71AV1, DBR2, and ALDH1 (Aldehyde Dehydrogenase 1) genes [65]. All the abovementioned TFs were found in our transcriptome (Table 7).

In order to figure out which TFs are involved in catalpol, acteoside, and carotenoid biosynthesis in C. grandiflora Benth, MYB TFs of which the $\log _{2}(\mathrm{FC})>4$ were selected for performing phylogenetic analysis with 168 MYBs from Arabidopsis thaliana. As a result, a total of 28 MYBs, including 16 upregulated and 12 downregulated, were screened out in leaf vs. root. In kiwifruit, $A d M Y B 7, A d M Y B 8$, $A d M Y B R 2$, and $A d M Y B R 3$ play important roles in regulating carotenoid accumulation in tissues through transcriptional activation of metabolic pathway genes $[35,66]$. In our analysis, CgMYB18, CgMYB26, CgMYB19, and AdMYB7 were all clustered into the S20 subgroup, while AdMYB8 was near the S20; CgMYB15, CgMYB4, CgMYB8, CgMYB13, AdMYBR2, and AdMYBR3 were in the same clade; and $C g M Y B 18, C g M Y B 26, C g M Y B 19, C g M Y B 15, C g M Y B 4, C g M Y B 8$, and $C g M Y B 13$ were all upregulated in the root, which indicates that they may regulate carotenoid biosynthesis in the roots of C. grandiflora Benth. (Figure 13a). In A. annua, overexpression of AaMYB1 exclusively in trichomes or in whole plants both increased the expression of the FDS (farnesyl diphosphate synthase), ADS, CYP71AV1, $D B R 2$, and $A L D H 1$ genes and increased the accumulation of artemisinin [67]. CgMYB9 and AaMYB1 were clustered into S13 subgroup and $C g M Y B 9$ was upregulated in leaves, which showed that it may be a candidate regulatory gene in catalpol and carotenoid biosynthesis [66]. Overexpression of AtPAP1 (Production of Anthocyanin Pigment1) in rose plants enhanced production of phenylpropanoid and terpenoid scent compounds by transcriptional activation of their respective pathway genes [68], and AtPAP1, AtMYB90 (AtPAP2), AtMYB113, and AtMYB114 all regulated anthocyanin biosynthesis [69]. In our data, CgMYB1, CgMYB2, CgMYB6, AtPAP1, and AtMYB90 were all in the S6 subgroup and $C g M Y B 1, C g M Y B 2$, and $C g M Y B 6$ were all significantly upregulated in the leaves (Figure 13b), suggesting that they are candidate regulatory genes in catalpol, acteoside, and carotenoid biosynthesis. 


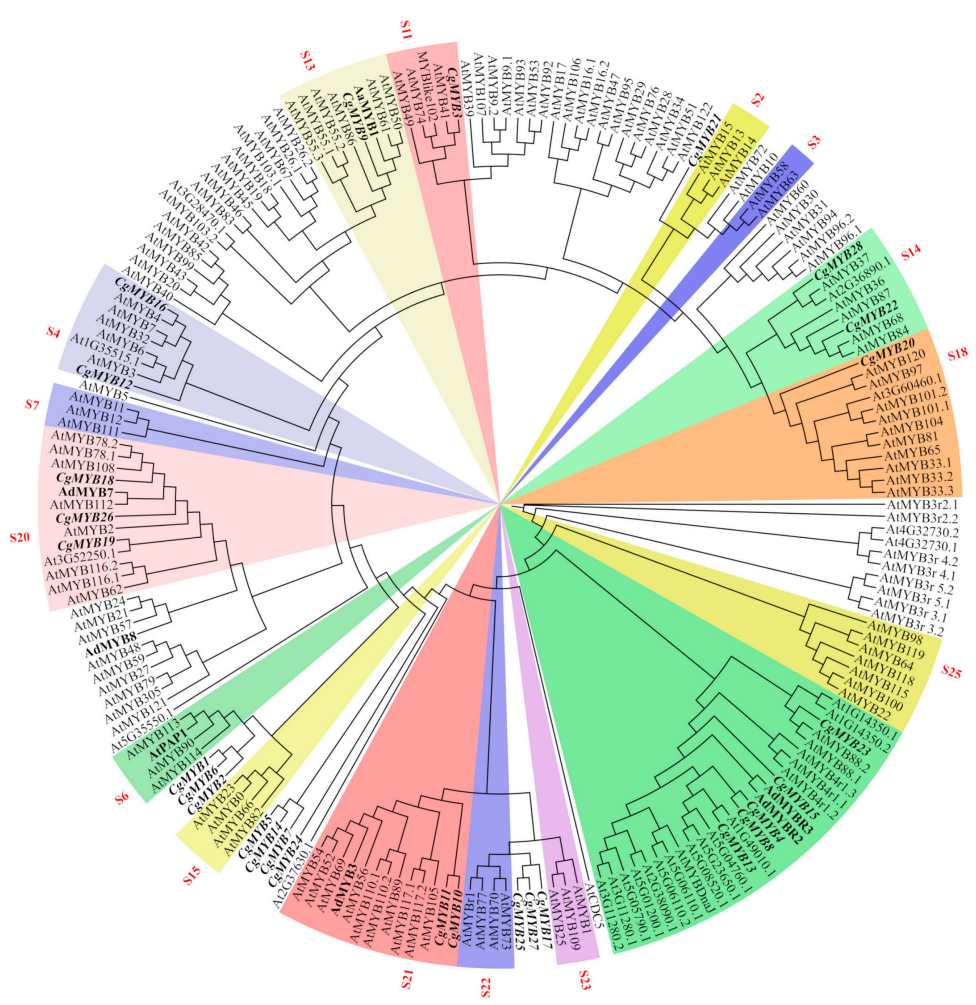

(a)

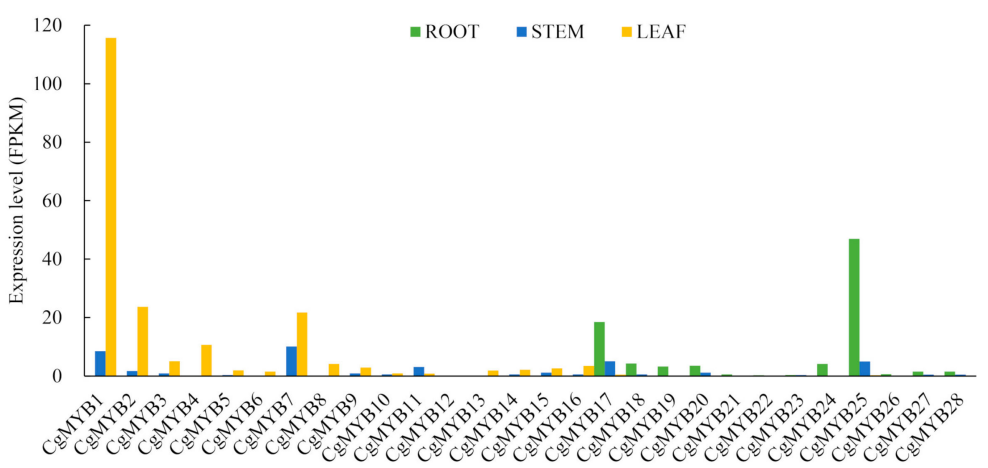

(b)

Figure 13. Phylogenetic analysis and expression level of MYBs from C. grandiflora Benth: (a) Phylogenetic analysis of CgMYBs. Amino acid sequences were aligned using the ClustalX2 program, and evolutionary distances were calculated using phyML software with the maximum likelihood statistical method. The sequences of C. grandiflora Benth are listed in Table S14. The sequences of Arabidopsis thaliana come from PLANTTFDB (https://planttfdb.cbi.pku.edu.cn), while that of Artemisia annua and Actinidia deliciosa come from NCBI (https://www.ncbi.nlm.nih.gov). (b) Expression level of CgMYBs: Expression level for each gene is represented by the average RPKM in roots, stems, and leaves.

\subsection{Expression Correlation Analysis of Selected Genes}

To verify our transcriptome results, five terpenoid-related genes including three upregulated genes (MCS, GES, and IS) and two downregulated genes (8HGO and HMGR2) in leaf vs. root were selected for correlation analysis. All the selected genes possessed the same expression trend although the expression levels varied between RNA-Seq and qRT-PCR, especially for the $8 H G O$ and HMGR2 genes (Figure 14a). The overall correlation coefficient was about 0.84 , which indicates that our transcriptome is valid (Figure 14b). 


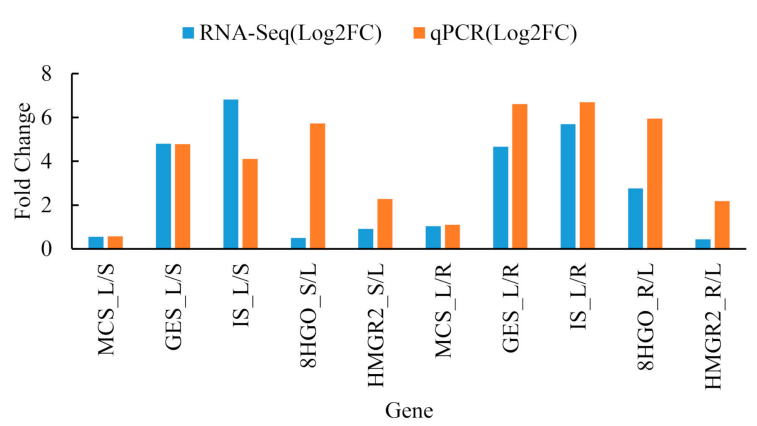

(a)

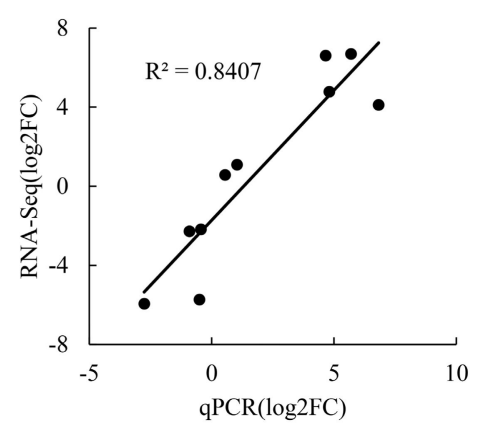

(b)

Figure 14. The correlation analysis of gene expression pattern between RNA-Seq and qPCR in roots, stems, and leaves in C. grandiflora Benth. (a) Expression patterns of MCS, GES, IS, 8HGO and HMGR2 gene by RNA-Seq and qPCR; (b) Correlation analysis of gene expression between RNA-Seq and qPCR. Each qPCR was biologically repeated three times.

\section{Discussion}

Cardiovascular diseases (CVDs) remain a major cause of health loss for all regions of the world in the past 25 years [70]. In China, the incidence of CVDs is continuously rising and will keep an upward trend in the next decade [2]. Therefore, to find the herbs with effective treatment of CVDs is imminent. C. grandiflora Benth is one of the most precious herbs in the area of Miao Nationality in Yunnan, China. It is widely used in folk medicine because of its multifunctional medicinal values, especially in the aspect of the prevention and treatment of CVDs [7]. Although it has been collected in the Chinese Materia Medica, up to now, it has not been included in the Chinese Pharmacopoeia because of limited researches [9]. The current situation is high market prices and overexploitation of wild resources, which has not only prevented the herbal medicine from being widely used but has destroyed species diversity. Synthetic biology will provide solutions for the abovementioned problems through the biosynthetic pathway elucidations of the main pharmacodynamic components.

So far, de novo transcriptome analysis is an important method in gene discovery of biosynthesis pathways, especially for species without reference genomes [13]. In this research, the transcriptomes of three tissues with three biological repeats were sequenced by illumine Hiseq2000, and 438,112,930 clean reads were assembled into 173,851 transcripts and 153,198 unigenes. This suggests that one gene may have different transcripts which may come from variable splicing, alleles, different copies of the same gene, homologs, orthologs, etc. The mean length of transcripts and genes were $1895 \mathrm{bp}$ and 2115 bp, respectively, and the N50 of the transcripts and genes were 2902 and 2936 bp, respectively (Table 2), which were higher than that in Dendrobium huoshanense, Persea Americana, and R. glutinosa [18,71,72]. These results implied that our assembly quality was suitable for subsequent analyses. In the species distribution analysis of unigenes, more than $43.77 \%$ of unigenes were matched to Sesamum indicum (Figure 2), which is similar to R. glutinosa, a plant of Scrophulariaceae; these results implied that they shared the closer genetic relationship, similar chemical substances, and similar biosynthetic pathways. Catalpol, acteoside, and azafrin are three medicinal ingredients in C. grandiflora Benth; however, their biosynthetic pathway is unexplored.

So far, catalpol biosynthesis containing terpenoid backbone pathway and iridoid pathway has not been fully deciphered due to the deficiency of detailed information on genetic and molecular levels [20]. In 1993, Damtoft found that 8-epi-deoxyloganic acid, bartsioside, and aucubin are intermediates of catalpol biosynthesis by feeding experiments [48]. Then, Jensen et al. confirmed that catalpol is synthesized via decarboxylated iridoids pathway (Route II), which involved 8-epi-iridodial, 8-epi-iridotrial, and 8-epi-deoxyloganic acid [73]. In 2013, the more detailed route II was proposed in $R$. glutinosa and P. kurrooa [21,41]. In 2015, the complete catalpol biosynthesis pathway was hypothesized in P. kurrooa according to data of the transcriptome mining, gene expression, and picroside content [17]. In our transcriptomes, 368 unigenes were annotated to the catalpol biosynthetic pathway with 
60 unigenes upregulated in leaves and 39 unigenes in roots; simultaneously combined with the fact that $F 3 D$ gene was not expressed in roots, we deduced that catalpol biosynthesis was mainly active in leaves. A recent article showed that, in wild C. grandiflora Benth, the content of catalpol is far higher in leaves than in stems and roots [74], which also implied that catalpol is mainly synthesized in leaves other than roots. The discovery of rate-limiting enzymes is essential for synthetic biology; therefore, some genes are discussed here. Catalpol biosynthesis begins with the terpenoid backbone pathway, which contains the MEP and MVA pathways. In the MEP pathway, the DXS enzyme is the first and rate-limiting enzyme, and in A. annua, among the three AaDXSs, only AaDXS2 might participate in artemisinin biosynthesis [75]. Contrary to A. annua, the DXSs were more abundant in our transcriptome, which seems that DXS was not a limiting enzyme in C. grandiflora Benth. Further studies are needed to clarify which DXS functions in MEP pathway. A recent report showed that plastidial IDI plays an important role in optimizing the ratio between IPP and DMADP as precursors for different downstream isoprenoid pathways while mutation of IDI1 reduced the content of carotenoids in fruits, flowers, and cotyledons (except mature leaves) [44]. In our transcriptome, there were 28 IDI genes with two upregulated in leaves compared with roots, which highlights their importance in terpenoid backbone biosynthesis (Table 4). However, there were no significant differences for the overall expression of IDI genes in roots, stems, and leaves in our transcriptome (Figure 9b). What is interesting is that there was only one MCS gene in our transcriptome; however, its expression levels in roots, stems, and leaves were all relatively high, which directly denied that MCS was a rate-limiting enzyme gene. According to the expression profile, $M C T$ may be a rate-limiting enzyme for roots (Figure 9b). In addition, the relative contribution of the MEP and MVA pathways for a specific pathway is a focus scientist paying attention to. In P. kurroa, the biosynthesis of picroside-I is contributed solely by the MEP pathway [17]. In Taxus baccata, the MEP pathway provides the main source of universal terpenoid precursor IPP [76]. However, in C. grandiflora Benth, the contribution of the MEP and MVA pathways for catalpol biosynthesis remains to be clarified and it will be resolved by the inhibition experiments in the future.

Acteoside biosynthesis was first studies in an O. europaea cell with feeding experiments, which outline the basic pathway profile: caffeoyl moiety was synthesized through the phenylalanine-derived pathway including intermediates cinnamic acid, p-coumaric acid, and caffeic acid, while hydroxytyrosol moiety was formed via the tyrosine-derived pathway including two alternative routes [26]. Then, HCT enzyme which connects the caffeoyl moiety and the hydroxytyrosol moiety, UGT enzymes, and the corresponding enzymes of the phenylalanine-derived pathway and tyrosine-derived pathway were hypothesized in R. glutinosa [28]. All of the acteoside pathway genes were found in our transcriptome of C. grandiflora Benth. Expression profiles showed that genes involved in both the phenylalanine-derived pathway and the tyrosine-derived pathway were more abundant in leaves and stems compared to roots, especially for the PAL and PPO genes (Figure 10b). This is consistent with the reports that, in Harpagophytum procumbens, the content of acteoside was higher in leaves and stems than in roots and that, in Sesamum indicum, the content of acteoside in leaves is far higher than in stems and roots [25,77].

Studies have shown that PAL is an entry-point enzyme which can convert $L$-Phe into trans-cinnamic acid and that it plays a vital role in channeling carbon flux from primary metabolism into the phenylpropanoid pathway [78]. So far, $P A L$ gene has been cloned from many medicinal plants, such as Ocimum basilicum [79], Ginkgo biloba [80], Salvia miltiorrhiza [81], and A. annua [82]. In G. biloba, the highest expression of GbPAL gene was found in leaves, followed by stems, and the lowest expression was in roots; transcription levels of GbPAL were closely related to flavonoid accumulation [80]. In $R$. glutinosa, the RgPAL gene (CL1389.Contig1) shared the same expression pattern as in G. biloba [28]. In $A$. апnua, the highest expression of the AaPAL gene was found in young leaves and the lowest expression of that was in roots [82]. In plants, $P A L$ gene is a multi-gene family and the gene number ranges from 4 in $A$. thaliana to more than 12 in tomato and potato [83]. For example, there are 6 PAL genes in R. glutinosa [28]. Recently, three different redundancy phenomena including active compensation in ligand plus passive compensation in receptor in tomato, passive compensation in 
ligand plus active compensation in receptor in Arabidopsis, and active compensation in both in corn have been figured out [84]; however, which type does the $C g P A L$ genes belong to and whether they benefit the plants themselves in C. grandiflora Benth remain to be discovered. Unlike potato, the PAL gene family is highly redundant but underutilized due to the highly silencing mechanism in tomato [83]. In our transcriptome, there are 19 PAL genes and their highest expressions are found in leaves and stems with the lowest expression in roots (Figure 10b), which is similar to that in G. biloba, R. glutinosa, and A. annua [28,80,82]. Our transcriptome profiling data showed that 10 of $19 \mathrm{CgPAL}$ genes were not expressed or slightly expressed in roots, stems, and leaves (Figure S1), which implied that gene silencing was also active in C. grandiflora Benth, and DNA cytosine methylation may account for this phenomenon [83]. A recent report showed that functional redundancy among $B Z R / B E H$ (BRASSINAZOLE-RESISTANT/BRI1-EMS-SUPRESSOR1/BRASSINAZOLE-RESISTANT1 HOMOLOG) gene family members is not necessary for trait robustness [85]. Even in tomato, only PAL5 was expressed under environmental stimuli [83]. Therefore, $P A L$ genes including the 3 significantly upregulated and 1 significantly downregulated in leaf vs. root in C. grandiflora Benth played important roles in acteoside biosynthesis (Table 5).

Polyphenol oxidase is usually undesirable in fruit and vegetable due to the browning, while it is desirable in tea, coffee, cocoa, etc. for the pigmentation [86]. Polyphenol oxidase (1,2-benzenediol: oxygen oxidoreductase), also known as tyrosinase, catechol oxidase, and laccase according to the specific substrate and reaction mechanism, is a group of copper-containing proteins $[86,87]$. A typical PPO protein contains three conservative regions: an $\mathrm{N}$-terminal transit peptide that is responsible for the import of PPO into the thylakoid lumen; a di-copper center, each with three histidine residues to bind a copper atom; and a C-terminal region [88]. Polyphenol oxidases can catalyze two quite different types of reactions: monophenol monooxygenases (E.C. 1.14.18.1) activity and o-diphenol oxidation reactions including catechol oxidases (E.C. 1.10.3.1) and laccases (E.C. 1.10.3.2) activity [87]. In plants, polyphenol oxidase is localized in chloroplasts and the reaction product accumulated in thylakoid [89]. The number of $P P O$ gene ranges from 1 to 13 in land plants with 0 for green algae and $A$. thaliana, and tandem duplications of the $P P O$ gene family is common in dicotyledon [88]. In our transcriptome, 11 PPO genes were clustered into three groups. Expression levels of the upper group including PPO7, PPO9, PPO10, and PPO11 were higher in leaves and stems compared with roots, while that of the bottom group including PPO1, $P P O 2$, and $P P O 3$ were higher in roots and stems than in leaves with the somewhat low expressions in middle group including PPO4, PPO5, PPO6, and PPO8 (Figure S2). Phylogenetic analysis of 11 CgPPOs with 6 PPOs of Solanum melongena and 6 PPOs of Solanum lycopersicum showed that all of our CgPPO proteins are clustered into one clade and that the other 12 PPO proteins formed another two clades (Figure S3). These species-specific PPO clades were also found in four major land plant lineages including Populus trichocarpa, Glycine max, Vitis vinifera, and Aquilegia coerulea, which implied that $C g P P O$ genes were also formed by independent burst of gene duplication [88].

Azafrin $\left(\mathrm{C}_{27} \mathrm{H}_{38} \mathrm{O}_{4}\right)$ derivates from tetraterpenoids $\left(\mathrm{C}_{40}\right)$. It has been found in many medicinal plants such as rhizome of Alectra chitrakutensis, Bergenia ciliate, Caralluma umbellate, and Alectra parasitica, and it has the functions of being antimicrobial, anti-inflammatory, analgesic, antioxidant, treatment of cardiovascular diseases [90-93]. Roots of C. grandiflora Benth display orange-yellow color, which is largely due to the presence of abundant azafrin as A. parasitica [93]. So far, the biosynthetic pathway of azafrin is not established from perspectives of chemistry and biology. There are studies implying that excentric cleavage of carotenoid compounds is a possible route [94]. CCD7 can catalyze $\beta$-carotene $\left(C_{40}\right)$ into $10^{\prime}$-apo- $\beta$-carotenal $\left(C_{27}\right)$ and ionone $\left(C_{13}\right)$ to support the above hypothesis [39]. In the view of molecular structure, the differences between $10^{\prime}$-apo- $\beta$-carotenal and azafrin are one terminal carboxyl group and two hydroxyl groups in cyclohexane skeleton. Therefore, two reactions are indispensable from $10^{\prime}$-apo- $\beta$-carotenal to azafrin: one is to convert the aldehyde group into carboxyl groups, and the other is to insert two oxygen atoms into cyclohexane skeleton to generate two hydroxyl groups. The ALDH superfamily comprises a group of enzymes involved 
in the $\mathrm{NAD}^{+}$(Nicotinamide Adenine Dinucleotide) or $\mathrm{NADP}^{+}$(Nicotinamide Adenine Dinucleotide Phosphate)-dependent conversion of various aldehydes to their corresponding carboxylic acids [95]. Although there are only $76 \mathrm{NAD}^{+}$-dependent $A L D H$ genes in our transcriptome, they are candidate genes for azafrin biosynthesis. In plant, CYP450s are responsible for many oxidative reactions such as hydroxylation, epoxidation, dealkylation, and dehydration, and the reactions catalyzed by CYP450s are irreversible [53]. There are 413 CYP450 unigenes in our transcriptome, of which 5 are significantly upregulated $\left(\log _{2}(\mathrm{FC})>10\right)$ and 5 are significantly downregulated in leaf vs. root (Table 6). They can be candidate genes of azafrin biosynthesis. The key enzymes determine the flux of the pathway, and the expression of the key enzyme gene dominates the number of enzymes. In marigold, the expression level of the LCYE gene in petals and $L C Y B$ gene in leaves were positively correlated with the lutein content [96]. In Momordica cochinchinensis, transcriptional regulation of genes including HMGR, HDS, PSY, PDS, ZDS, CRTISO, and LCYE may determine the alteration of carotenoid content during fruit ripening [97]. Our transcriptome data showed that only the expression levels of HDS, PSY, ZDS, and CRTISO were more abundant in roots than leaves and stems (Figure 11b). However, trace expression of the DWARF27 gene in leaves and low expression of the CCD7 gene in roots, stems, and leaves suggested that they were two rate-limiting enzymes in azafrin biosynthesis. DWARF27, which exhibits increased tillers and reduced plant height, was first studied in rice [98]. It encodes an iron-containing protein localized in chloroplasts and is expressed mainly in vascular cells of shoots and roots [98]. Further studies indicated that DWARF27 is an all-trans/9-cis isomerase which can convert all-trans- $\beta$-carotene into 9-cis- $\beta$-carotene in vivo and in vitro [99]. Obviously, DWARF27 is vital for azafrin biosynthesis. What is interesting is that there is only one $C C D 7$ gene in our transcriptome which coincides with the all CCD7 genes identified including maize, rice, sorghum, Selaginella moellendorfii, Physcomitrella patens, and Chlamydomonas reinhardtii and is a single copy [100]. The highest expression of the CCD7 gene was found in roots among maize, A. thaliana, pea, and petunia [100]. However, the highest expression in our transcriptome is in stems.

In the future, studies related to catalpol, acteoside, and azafrin biosynthesis will focus on the following aspects: (1) to construct a transgenic system for $C$. grandiflora Benth according to the successive tissue culture technology for verification of gene function, to characterize the putative genes of three pathways, and to verify their functions by enzyme assays in vitro or to overexpress them in vivo; (2) to explore the correlation between the contents of active component and related gene expression levels, to clone the putative TFs, and to verify their functions in the biosynthesis of active components via chromatin immunoprecipitation and overexpression in vivo; and (3) to figure out the biosynthetic pathway using feeding experiments with suspension cells.

\section{Materials and Methods}

\subsection{Plant Materials and RNA Isolation}

The artificial, cultivated Centranthera grandiflora Benth was grown in fields with Cyperus rotundus in Yushancheng base, Yuxi Flyingbear Agricultural Development Company Limited, Yuxi, Yunnan Province, China (Figure 15a). Three healthy plants with the same growth potential were selected, and the fresh roots, stems, and leaves were collected from one-year-old C. grandiflora plants on May 7, 2018 (Figure 15b). Materials from three individual plants were collected using scissors to yield $1 \mathrm{~g}$ of root, stem, and leaf samples (BioSample accessions: SAMN12499651, SAMN12499652, SAMN12499653, SAMN12499654, SAMN12499655, SAMN12499656, SAMN12499657, SAMN12499658, and SAMN12499659). After wrapping with tinfoil and tagging, all samples were immediately frozen in liquid nitrogen and stored at $-80^{\circ} \mathrm{C}$. 


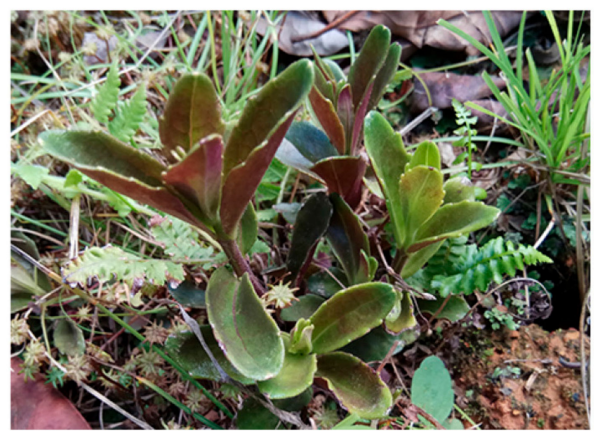

(a)

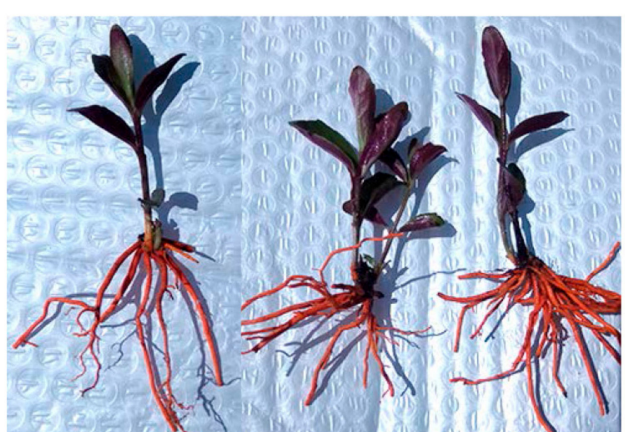

(b)

Figure 15. Plant materials of C. grandiflora Benth: (a) C. grandiflora Benth growing in the field with Cyperus rotundus and (b) Roots, stems, and leaves used in experiments for sequencing and qRT-PCR.

For total RNA extraction and quality control, refer to Zhang et al. [101].

\subsection{Library Preparation for Transcriptome Sequencing}

A total amount of $1.5 \mu \mathrm{g}$ RNA per sample was used as input material for the RNA sample preparations. Sequencing libraries were generated using the NEBNext ${ }^{\circledR}$ UItra $^{\mathrm{TM}}$ RNA Library Prep Kit for Illumina (NEB, Ipswich, MA, USA) following the manufacturer's recommendations. Index codes were added to attribute sequences to each sample. Briefly, mRNA was purified from total RNA using poly-T oligo-attached magnetic beads. Fragmentation was carried out using divalent cations under elevated temperature in a NEBNext First Strand Synthesis Reaction Buffer (5×). First strand cDNA was synthesized using random hexamer primer and M-MuLV Reverse Transcriptase (RNase $\mathrm{H}^{-}$). Second-strand cDNA synthesis was subsequently performed using DNA Polymerase I and RNase $H$. Remaining overhangs were converted into blunt ends via exonuclease/polymerase activities. After adenylation of 3' ends of DNA fragments, NEBNext Adaptor with hairpin loop structure was ligated to prepare for hybridization. In order to select cDNA fragments preferentially 250-300 bp in length, the library fragments were purified with AMPure XP system (Beckman Coulter, Beverly, USA). Then, $3 \mu \mathrm{L}$ USER Enzyme (NEB, Ipswich, MA, USA) was used with size-selected, adaptor-ligated cDNA at $37^{\circ} \mathrm{C}$ for $15 \mathrm{~min}$ followed by $5 \mathrm{~min}$ at $95^{\circ} \mathrm{C}$ before PCR. PCR was performed with Phusion High-Fidelity DNA polymerase, Universal PCR primers, and Index $(X)$ Primer. At last, $P C R$ products were purified (AMPure XP system) and library quality was assessed on the Agilent Bioanalyzer 2100 system (Agilent Technologies, Palo Alto, CA, USA).

\subsection{Clustering and Sequencing}

The clustering of the index-coded samples was performed on a cBot Cluster Generation System using TruSeq PE Cluster Kit v3-cBot-HS (Illumia, San Diego, CA, USA) according to the manufacturer's instructions. After cluster generation, the library preparations were sequenced on an Illumina Hiseq2000 platform and paired-end reads were generated.

\subsection{Data Filtering and Transcriptome Assembly}

The flow of bioinformatics analysis is showed in Figure 16. Before assembly, raw reads containing adaptors, more than $10 \% \mathrm{~N}$ bases, and more than $50 \%$ of low quality were removed to obtain clean reads. Meanwhile, Q20, Q30, and GC content were used to assess the data quality. All the subsequent analyses were based on these clean reads. As there are no reference genomes available for $C$. grandiflora, the clean reads of roots, stems, and leaves were assembled together. The paired-end reads of each sample were merged into one interleaved fastq file. All the nine pooled files were assembled using Trinity software (version: r20140413p1) (Cambridge, MA, USA) with min_k-mer_cov set to 2 and all 
other parameters settings as default [102]. After clustering and de-redundancy by Corset software (version: 1.07) (VIC, Austrilia) [103], the clean nonredundant unigenes was generated.

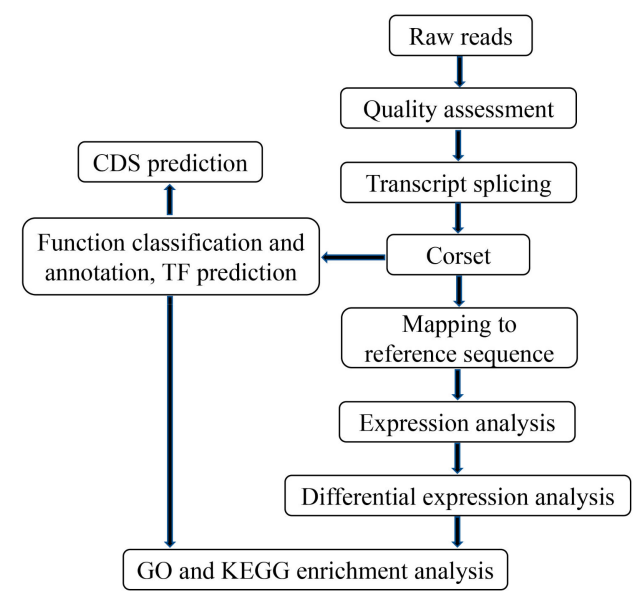

Figure 16. Flow chart of transcriptome bioinformatics analysis for C. grandiflora Benth.

\subsection{Gene Functional Annotation}

Gene function was annotated based on the following databases: $\mathrm{Nr}$ (NCBI nonredundant protein sequences, diamond v0.8.22, $e$-value $=10^{-5}$ ), NT (NCBI nucleotide sequences, NCBI blast 2.2.28+, $e$-value $=10^{-5}$ ), PFAM (Protein family, HMMER 3.0 package, hmmscan $e$-value $=10^{-2}$ ), SwissProt (a manually annotated and reviewed protein sequence database, diamond v0.8.22, $e$-value $=10^{-5}$ ), KOG/COG (Clusters of Orthologous Groups of proteins/euKaryotic Ortholog Groups, diamond v0.8.22, $e$-value $=10^{-5}$ ), KAAS (version: r140224, $e$-value $=10^{-10}$ ), and GO (Blast2GO v2.5 and inhouse script, $e$-value $\left.=10^{-6}\right)$. To figure out the TF families involved in the active ingredient biosynthesis, iTAK software (https://github.com/kentn/iTAK/) was used to predict the TF. Its basic principle is to identify TF by hmmscan using TF family and rules defined by classification in the database. For the identification and classification methods of TF, refer to Perez-Rodriguez et al. [104].

\subsection{Differential Expression Analysis}

The calculation of unigene expression was performed using the RPKM method, and gene expression levels were estimated by RSEM (version: 1.2.15, parameter for bowtie2: mismatch 0) for each sample [43]. Differential expression analysis of two organs was performed using the DESeq R package 1.10.1. DESeq provides statistical routines for determining differential expression in digital gene expression data using a model based on the negative binomial distribution. The resulting $p$ values were adjusted using Benjamini and Hocberg's approach for controlling false discovery rates. Genes with a threshold of foldchange $\geq 2$ and $p$-value $<0.05$ found by DESeq were assigned as differentially expressed.

\subsection{GO Enrichment and KEGG Pathway Enrichment Analysis}

Gene ontology enrichment analysis of the differentially expressed genes (DEGs) was implemented by the GOseq (version: 1.10.0) and topGO (version: 2.10.0) R packages based Wallenius non-central hyper-genometric distribution, which can adjust for gene length bias in DEGs [105]. KOBAS software (Beijing, China) was used to test the statistical enrichment of DEGs in KEGG pathways [106]. The expressed genes $($ FPKM $\geq 1)$ were used as background with a corrected $p$-value $\leq 0.05$ for both enrichment analyses. 


\section{8. $q R T-P C R$ Analysis}

Total RNA was extracted from roots, stems, and leaves of annual C. grandiflora Benth. The first strand of DNA was synthesized using reverse transcription kit PrimeScript RT Master Mix (Perfect Real Time) (Takara, Dalian, China). Specific primers were designed according to the selected gene sequences for expression analysis (Table S14). Using the C. grandiflora Benth CgUbi gene (Accession number: MK256646) as an internal reference, qPCR was performed using chimeric fluorescence detection kit TB Green Premix Ex Taq II (Takara, China). Each reaction was repeated three times. Reaction was amplified by LightCycler 480II fluorescent quantitative PCR (Roche, Basel, Switzerland). After the amplification, results were calibrated by internal reference gene and the relative gene expressions in roots, stems, and leaves were calculated automatically by the $2^{-\Delta \Delta C t}$ method.

\subsection{Data Submission}

This Transcriptome Shotgun Assembly project (PRJNA558809) has been deposited at DDBJ/ENA/GenBank under the accession GHUX00000000. The version described in this paper is the first version, GHUX01000000.

\section{Conclusions}

Centranthera grandiflora Benth has been used to prevent and treat CVDs for a long time; however, the biosynthesis pathway of its active components including catalpol, acteoside, and azafrin remains undeciphered. Transcriptome sequencing technology is an effective way to discover the genes of this herb's biosynthesis pathways and its regulatory mechanisms. In this study, nine cDNA libraries were constructed from the roots, stems, and leaves of $C$. grandiflora Benth and sequenced by an Illumina Hiseq2000 platform. As a result, 438,112,930 clean reads were obtained and 153,198 unigenes were assembled. Among these genes, 557, 213, and 161 unigenes were annotated into catalpol, acteoside, and azafrin biosynthetic pathways, respectively. Azafrin can be synthesized through $\beta$-carotene, 9-cis- $\beta$-carotene, and $10^{\prime}$-apo- $\beta$-carotenal with the corresponding enzymes DWARF27, CCD7, ALDH, and CYP450. Also, the PAL gene silencing phenomenon is discovered and discussed. The candidate TF MYBs involved in the regulation of these pathways were proposed. Our results represent the first genomic resource for C. grandiflora Benth, which is a starting point for exploration of this valuable herb in molecular biology.

Supplementary Materials: Supplementary materials can be found at http://www.mdpi.com/1422-0067/20/23/ 6034/s1.

Author Contributions: X.Z. conceived and design of experiments. X.Z., C.L., and L.W. performed the experiments. X.Z., C.L., and W.Q. analyzed the data. Y.F. contributed materials. X.Z. wrote the manuscript, and W.Q. reviewed and edited the manuscript.

Funding: This research was funded by Yunnan Provincial Science and Technology Department (grant number 2017FH001-024, 2016FD113) and China Scholarship Council (grant number 201908530057).

Acknowledgments: We gratefully acknowledge Beijing Novogene Bioinformatics Technology Company of China for carrying out the sequencing of the transcriptomes. We gratefully acknowledge the anonymous reviewers for their constructive comments.

Conflicts of Interest: The authors declare no conflict of interest.

\section{References}

1. Clark, H. NCDs: A challenge to sustainable human development. Lancet 2013, 381, 510-511. [CrossRef]

2. Chen, W.; Gao, R.; Liu, L.; Zhu, M.; Wang, W.; Wang, Y.; Wu, Z.; Li, H.; Gu, D.; Yang, Y. China cardiovascular diseases report 2015: A summary. J. Geriatr. Cardiol. 2017, 14, 1-10. [CrossRef] [PubMed]

3. Hao, P.; Jiang, F.; Cheng, J.; Ma, L.; Zhang, Y.; Zhao, Y. Traditional Chinese medicine for cardiovascular disease: Evidence and potential mechanisms. J. Am. Coll. Cardiol. 2017, 69, 2952-2966. [CrossRef] [PubMed]

4. Editorial Committee of Flora of China. Flora of China, 1st ed.; Science Press: Beijing, China, 1979; p. 345. 
5. Liang, D. A breakthrough in tissue culture and rapid propagation of rare and endangered medicinal Centranthera grandiflora Benth. Yunnan Information Daily, 14 May 2018.

6. Liao, L.; Zhang, Z.; Hu, Z.; Chou, G.; Wang, Z. Iridoid glycosides from Centranthera grandiflora. Chin. Tradit. Herb. Drugs 2012, 43, 2369-2371.

7. Liao, L. Investigation into the Bioactive Components and Chemical Constituents from the Roots of Centranthera grandijlora Benth. Ph.D. Thesis, Shanghai University of Traditional Chinese Medicine, Shanghai, China, 21 June 2014.

8. Liang, J.; Zhang, J.; Ma, X.; Wang, G.; Chen, Y.; Wen, Y.; Gan, L. Identification of chemical constituents from Centranthera grandiflora. Chin. Bull. Bot. 1984, 2, 47.

9. Editorial Committee of Chinese Materia Medica. Chinese Materia Medica, 1st ed.; Shanghai Science and Technology Press: Shanghai, China, 1996; p. 397.

10. Wang, Z.; Wang, Q.; Yan, J.; Cong, Y. Chemical constituents and activities research of Centranthera grandiflora Benth. In Proceedings of the Yunnan Pharmaceutical Conference in 2012, Yunnan Pharmaceutical Conference in 2012, Kunming, China, 20 September 2012; pp. 1-4.

11. Chen, M.; Ye, Z.; Xueying, Z.; Jianjun, Z.; Yongchun, Z.; Liqing, Y.; Liuyan, Y. A tissue culture method of Centranthera grandiflora Benth. China Patent ZL201710499456.0, 15 March 2019.

12. Hua, W.; Zheng, P.; He, Y.; Cui, L.; Kong, W.; Wang, Z. An insight into the genes involved in secoiridoid biosynthesis in Gentiana macrophylla by RNA-seq. Mol. Biol. Rep. 2014, 41, 4817-4825. [CrossRef]

13. Liu, Y.; Wang, Y.; Guo, F.; Zhan, L.; Mohr, T.; Cheng, P.; Huo, N.; Gu, R.; Pei, D.; Sun, J.; et al. Deep sequencing and transcriptome analyses to identify genes involved in secoiridoid biosynthesis in the Tibetan medicinal plant Swertia mussotii. Sci. Rep. 2017, 7, 43108. [CrossRef]

14. Miettinen, K.; Dong, L.; Navrot, N.; Schneider, T.; Burlat, V.; Pollier, J.; Woittiez, L.; van der Krol, S.; Lugan, R.; Ilc, T.; et al. The seco-iridoid pathway from Catharanthus roseus. Nat. Commun. 2014, 5, 3606-3616. [CrossRef]

15. Munkert, J.; Pollier, J.; Miettinen, K.; Van Moerkercke, A.; Payne, R.; Müller-Uri, F.; Burlat, V.; O'Connor, S.E.; Memelink, J.; Kreis, W. Iridoid synthase sctivity is common among the plant progesterone $5 \beta$-reductase family. Mol. Plant 2015, 8, 136-152. [CrossRef]

16. Huang, L.; Liu, C. Molecular Pharmacognosy, 3rd ed.; Science Press: Beijing, China, 2015; pp. $238-342$.

17. Shitiz, K.; Sharma, N.; Pal, T.; Sood, H.; Chauhan, R.S. NGS transcriptomes and enzyme inhibitors unravel complexity of picrosides biosynthesis in Picrorhiza kurroa Royle ex. Benth. Plos ONE 2015, 10, e0144546. [CrossRef] [PubMed]

18. Zhi, J.; Li, Y.; Zhang, Z.; Yang, C.; Xie, C. Molecular regulation of catalpol and acteoside accumulation in radial striation and non-radial striation of Rehmannia glutinosa tuberous root. Int. J. Mol. Sci. 2018, 19, 3751. [CrossRef] [PubMed]

19. Xue, L.; He, Z.; Bi, X.; Xu, W.; Wei, T.; Wu, S.; Hu, S. Transcriptomic profiling reveals MEP pathway contributing to ginsenoside biosynthesis in Panax ginseng. BMC Genom. 2019, 20, 134. [CrossRef] [PubMed]

20. Dinda, B. Chemistry and Biosynthesis of Iridoids. In Pharmacology and Applications of Naturally Occurring Iridoids, 1st ed.; Dinda, B., Ed.; Springer International Publishing: Cham, Switzerland, 2019; pp. 119-143.

21. Wajid, W.B.; Niha, D.; Sumeer, R.; Satiander, R.; Rukmankesh, M.; Amit, N.; Rekha, S.D.; Nasheeman, A.; Ram, V.; Surrinder, K.L. Molecular characterization of UGT94F2 and UGT86C4, two glycosyltransferases from Picrorhiza kurrooa: Comparative structural insight and evaluation of substrate recognition. PLoS ONE 2013, 8, e73804. [CrossRef]

22. Kumar, V. OMICS-based approaches for elucidation of picrosides bsynthesis in Picrorhiza kurroa. In OMICS-Based Approaches in Plant Biotechnology, 1st ed.; Banerjee, R., Kumar, V., Kumar, J., Eds.; Scrivener Publishing LLC: Beverly, MA, USA, 2019; pp. 145-166.

23. Ye, P.; Liang, S.; Wang, X.; Duan, L.; Jiang-Yan, F.; Yang, J.; Zhan, R.; Ma, D. Transcriptome analysis and targeted metabolic profiling for pathway elucidation and identification of a geraniol synthase involved in iridoid biosynthesis from Gardenia jasminoides. Ind. Crop. Prod. 2019, 132, 48-58. [CrossRef]

24. Nagatoshi, M.; Terasaka, K.; Nagatsu, A.; Mizukami, H. Iridoid-specific Glucosyltransferase from Gardenia jasminoides. J. Biol. Chem. 2011, 286, 32866-32874. [CrossRef]

25. Fuji, Y.; Ohtsuki, T.; Matsufuji, H. Accumulation and subcellular localization of acteoside in sesame plants (Sesamum indicum L.). ACS Omega 2018, 3, 17287-17294. [CrossRef]

26. Saimaru, H.; Orihara, Y. Biosynthesis of acteoside in cultured cells of Olea europaea. J. Nat. Med. 2010, 64, 139-145. [CrossRef] 
27. Alipieva, K.; Korkina, L.; Orhan, I.E.; Georgiev, M.I. Verbascoside-A review of its occurrence, (bio)synthesis and pharmacological significance. Biotechnol. Adv. 2014, 32, 1065-1076. [CrossRef]

28. Wang, F.; Zhi, J.; Zhang, Z.; Wang, L.; Suo, Y.; Xie, C.; Li, M.; Zhang, B.; Du, J.; Gu, L. Transcriptome analysis of salicylic acid treatment in Rehmannia glutinosa hairy roots using RNA-seq technique for identification of genes involved in acteoside biosynthesis. Front. Plant Sci. 2017, 8, 787. [CrossRef]

29. Cheimonidi, C.; Samara, P.; Polychronopoulos, P.; Tsakiri, E.N.; Nikou, T.; Myrianthopoulos, V.; Sakellaropoulos, T.; Zoumpourlis, V.; Mikros, E.; Papassideri, I. Selective cytotoxicity of the herbal substance acteoside against tumor cells and its mechanistic insights. Redox Biol. 2018, 16, 169-178. [CrossRef] [PubMed]

30. Deng, H.; Sun, M.; Chen, H.; Wang, X.; Qiong, W.; Chang, Q. Effect of acteoside on behavioral changes and endoplasmic reticulum stress in prefrontal cortex of depressive rats. Chin. J. Pathophysiol. 2018, 34, 101-106.

31. Khullar, M.; Sharma, A.; Wani, A.; Sharma, N.; Sharma, N.; Chandan, B.; Kumar, A.; Ahmed, Z. Acteoside ameliorates inflammatory responses through NFkB pathway in alcohol induced hepatic damage. Int. Immunopharmacol. 2019, 69, 109-117. [CrossRef]

32. Bai, Y.; Zhu, R.; Tian, Y.; Li, R.; Chen, B.; Zhang, H.; Xia, B.; Zhao, D.; Mo, F.; Zhang, D. Catalpol in diabetes and its complications: A review of pharmacology, pharmacokinetics, and safety. Molecules 2019, 24, 3302. [CrossRef] [PubMed]

33. Yang, S.; Chou, G.; Li, Q. Cardioprotective role of azafrin in against myocardial injury in rats via activation of the Nrf2-ARE pathway. Phytomedicine 2018, 47, 12-22. [CrossRef] [PubMed]

34. Ohmiya, A.; Kato, M.; Shimada, T.; Nashima, K.; Kishimoto, S.; Nagata, M. Molecular basis of carotenoid accumulation in horticultural crops. Horticult. J. 2019, UTD-R003. [CrossRef]

35. Ampomah-Dwamena, C.; Thrimawithana, A.H.; Dejnoprat, S.; Lewis, D.; Espley, R.V.; Allan, A.C. A kiwifruit (Actinidia deliciosa) R2R3-MYB transcription factor modulates chlorophyll and carotenoid accumulation. New Phytol. 2019, 221, 309-325. [CrossRef]

36. Wang, Q.; Cao, T.; Zheng, H.; Zhou, C.; Wang, Z.; Wang, R.; Lu, S. Manipulation of carotenoid metabolic flux by lycopene cyclization in ripening red pepper (Capsicum annuum var. conoides) fruits. J. Agric. Food Chem. 2019, 67, 4300-4310. [CrossRef]

37. Kanehisa, M. Kegg Pathway Database. Available online: https://www.kegg.jp/kegg/pathway.html (accessed on 1 July 2019).

38. Schliemann, W.; Kolbe, B.; Schmidt, J.; Nimtz, M.; Wray, V. Accumulation of apocarotenoids in mycorrhizal roots of leek (Allium porrum). Phytochemistry 2008, 69, 1680-1688. [CrossRef]

39. Alder, A.; Jamil, M.; Marzorati, M.; Bruno, M.; Vermathen, M.; Bigler, P.; Ghisla, S.; Bouwmeester, H.; Beyer, P.; Al-Babili, S. The path from $\beta$-carotene to carlactone, a strigolactone-like plant hormone. Science 2012, 335, 1348-1351. [CrossRef]

40. Grabherr, M.G.; Haas, B.J.; Yassour, M.; Levin, J.Z.; Thompson, D.A.; Amit, I.; Adiconis, X.; Fan, L.; Raychowdhury, R.; Zeng, Q. Full-length transcriptome assembly from RNA-Seq data without a reference genome. Nat. Biotechnol. 2011, 29, 644-652. [CrossRef] [PubMed]

41. Sun, P.; Song, S.; Zhou, L.; Zhang, B.; Qi, J.; Li, X. Transcriptome analysis reveals putative genes involved in iridoid biosynthesis in Rehmannia glutinosa. Int. J. Mol. Sci. 2012, 13, 13748-13763. [CrossRef] [PubMed]

42. Cao, H.; Nuruzzaman, M.; Xiu, H.; Huang, J.; Wu, K.; Chen, X.; Li, J.; Wang, L.; Jeong, J.H.; Park, S.J.; et al. Transcriptome analysis of methyl jasmonate-elicited Panax ginseng adventitious roots to discover putative ginsenoside biosynthesis and transport genes. Int. J. Mol. Sci. 2015, 16, 3035-3057. [CrossRef] [PubMed]

43. Li, B.; Dewey, C. RSEM: Accurate transcript quantification from RNA-Seq data with or without a reference genome. BMC Bioinform. 2011, 12, 323. [CrossRef] [PubMed]

44. Pankratov, I.; McQuinn, R.; Schwartz, J.; Bar, E.; Fei, Z.; Lewinsohn, E.; Zamir, D.; Giovannoni, J.J.; Hirschberg, J. Fruit carotenoid-deficient mutants in tomato reveal a function of the plastidial isopentenyl diphosphate isomerase (IDI1) in carotenoid biosynthesis. Plant J. 2016, 88, 82-94. [CrossRef] [PubMed]

45. Wieffering, J. Aucubinartige glucoside (pseudoindikane) und verwandte heteroside als systematische merkmale. Phytochemistry 1966, 5, 1053-1064. [CrossRef]

46. Oshio, H.; Inouye, H. Iridoid glycosides of Rehmannia glutinosa. Phytochemistry 1982, 21, 133-138. [CrossRef]

47. Damtoft, S. Biosynthesis of the iridoids aucubin antirrinoside from 8-epi-deoxyloganic acid. Phytochemistry 1983, 22, 1929-1930. [CrossRef]

48. Damtoft, S. Biosynthesis of catalpol. Phytochemistry 1994, 35, 1187-1189. [CrossRef] 
49. He, J.; Hu, X.; Zeng, Y.; Li, Y.; Wu, H.; Qiu, R.; Ma, W.; Li, T.; Li, C.; He, Z. Advanced research on acteoside for chemistry and bioactivities. J. Asian Nat. Prod. Res. 2011, 13, 449-464. [CrossRef]

50. Berry, H.M.; Rickett, D.V.; Baxter, C.J.; Enfissi, E.M.A.; Fraser, P.D. Carotenoid biosynthesis and sequestration in red chilli pepper fruit and its impact on colour intensity traits. J. Exp. Bot. 2019, 70, 2637-2650. [CrossRef] [PubMed]

51. Kanehisa, M.; Goto, S.; Sato, Y.; Furumichi, M.; Tanabe, M. KEGG for integration and interpretation of large-scale molecular data sets. Nucleic Acids Res. 2011, 40, D109-D114. [CrossRef] [PubMed]

52. Dela Seña, C.; Sun, J.; Narayanasamy, S.; Riedl, K.M.; Yuan, Y.; Curley, R.W.; Schwartz, S.J.; Harrison, E.H. Substrate specificity of purified recombinant chicken $\beta$-carotene $9^{\prime}, 10^{\prime}$-oxygenase (BCO2). J. Biol. Chem. 2016, 291, 14609-14619. [CrossRef] [PubMed]

53. Rasool, S.; Mohamed, R. Plant cytochrome P450s: Nomenclature and involvement in natural product biosynthesis. Protoplasma 2016, 253, 1197-1209. [CrossRef] [PubMed]

54. Kirby, J.; Keasling, J.D. Biosynthesis of plant isoprenoids: Perspectives for microbial engineering. Annu. Rev. Plant Biol. 2009, 60, 335-355. [CrossRef]

55. Yan, T.; Chen, M.; Shen, Q.; Li, L.; Fu, X.; Pan, Q.; Tang, Y.; Shi, P.; Lv, Z.; Jiang, W. HOMEODOMAIN PROTEIN 1 is required for jasmonate-mediated glandular trichome initiation in Artemisia annua. New Phytol. 2017, 213, 1145-1155. [CrossRef]

56. Chen, M.; Yan, T.; Shen, Q.; Lu, X.; Pan, Q.; Huang, Y.; Tang, Y.; Fu, X.; Liu, M.; Jiang, W.; et al. GLANDULAR TRICHOME-SPECIFIC WRKY 1 promotes artemisinin biosynthesis in Artemisia annua. New Phytol. 2017, 214, 304-316. [CrossRef]

57. Tan, H.; Xiao, L.; Gao, S.; Li, Q.; Chen, J.; Xiao, Y.; Ji, Q.; Chen, R.; Chen, W.; Zhang, L. TRICHOME AND ARTEMISININ REGULATOR 1 is required for trichome development and artemisinin biosynthesis in Artemisia annua. Mol. Plant 2015, 8, 1396-1411. [CrossRef]

58. Yu, Z.; Li, J.; Yang, C.; Hu, W.; Wang, L.; Chen, X. The jasmonate-responsive AP2/ERF transcription factors AaERF1 and AaERF2 positively regulate artemisinin biosynthesis in Artemisia annua L. Mol. Plant 2012, 5 , 353-365. [CrossRef]

59. Han, J.; Wang, H.; Lundgren, A.; Brodelius, P.E. Effects of overexpression of AaWRKY1 on artemisinin biosynthesis in transgenic Artemisia annua plants. Phytochemistry 2014, 102, 89-96. [CrossRef]

60. Lu, X.; Zhang, L.; Zhang, F.; Jiang, W.; Shen, Q.; Zhang, L.; Lv, Z.; Wang, G.; Tang, K. AaORA, a trichome-specific AP2/ERF transcription factor of Artemisia annua, is a positive regulator in the artemisinin biosynthetic pathway and in disease resistance to Botrytis cinerea. New Phytol. 2013, 198, 1191-1202. [CrossRef] [PubMed]

61. Ji, Y.; Xiao, J.; Shen, Y.; Ma, D.; Li, Z.; Pu, G.; Li, X.; Huang, L.; Liu, B.; Ye, H.; et al. Cloning and characterization of AabHLH1, a bHLH transcription factor that positively regulates artemisinin biosynthesis in Artemisia annua. Plant Cell Physiol. 2014, 55, 1592-1604. [CrossRef] [PubMed]

62. Zhang, F.; Fu, X.; Lv, Z.; Lu, X.; Shen, Q.; Zhang, L.; Zhu, M.; Wang, G.; Sun, X.; Liao, Z. A basic leucine zipper transcription factor, AabZIP1, connects abscisic acid signaling with artemisinin biosynthesis in Artemisia annua. Mol. Plant 2015, 8, 163-175. [CrossRef] [PubMed]

63. Majid, I.; Kumar, A.; Abbas, N. A basic helix loop helix transcription factor, AaMYC2-Like positively regulates artemisinin biosynthesis in Artemisia annua L. Ind. Crop Prod. 2019, 128, 115-125. [CrossRef]

64. Shen, Q.; Lu, X.; Yan, T.; Fu, X.; Lv, Z.; Zhang, F.; Pan, Q.; Wang, G.; Sun, X.; Tang, K. The jasmonate-responsive AaMYC2 transcription factor positively regulates artemisinin biosynthesis in Artemisia annua. New Phytol. 2016, 210, 1269-1281. [CrossRef]

65. Shen, Q.; Zhang, L.; Liao, Z.; Wang, S.; Yan, T.; Shi, P.; Liu, M.; Fu, X.; Pan, Q.; Wang, Y. The genome of Artemisia annua provides insight into the evolution of Asteraceae family and artemisinin biosynthesis. Mol. Plant 2018, 11, 776-788. [CrossRef]

66. Dubos, C.; Stracke, R.; Grotewold, E.; Weisshaar, B.; Martin, C.; Lepiniec, L. MYB transcription factors in Arabidopsis. Trends Plant Sci. 2010, 15, 573-581. [CrossRef]

67. Matías-Hernández, L.; Jiang, W.; Yang, K.; Tang, K.; Brodelius, P.E.; Pelaz, S. AaMYB1 and its orthologue AtMYB61 affect terpene metabolism and trichome development in Artemisia annua and Arabidopsis thaliana. Plant J. 2017, 90, 520-534. [CrossRef] 
68. Zvi, M.M.B.; Shklarman, E.; Masci, T.; Kalev, H.; Debener, T.; Shafir, S.; Ovadis, M.; Vainstein, A. PAP1 transcription factor enhances production of phenylpropanoid and terpenoid scent compounds in rose flowers. New Phytol. 2012, 195, 335-345. [CrossRef]

69. Gonzalez, A.; Zhao, M.; Leavitt, J.M.; Lloyd, A.M. Regulation of the anthocyanin biosynthetic pathway by the TTG1/bHLH/Myb transcriptional complex in Arabidopsis seedlings. Plant J. 2008, 53, 814-827. [CrossRef]

70. Roth, G.A.; Johnson, C.; Abajobir, A.; Abd-Allah, F.; Abera, S.F.; Abyu, G.; Ahmed, M.; Aksut, B.; Alam, T.; Alam, K. Global, regional, and national burden of cardiovascular diseases for 10 causes, 1990 to 2015. J. Am. Coll. Cardiol. 2017, 70, 1-25. [CrossRef] [PubMed]

71. Yuan, Y.; Yu, M.; Jia, Z.; Song, X.; Liang, Y.; Zhang, J. Analysis of Dendrobium huoshanense transcriptome unveils putative genes associated with active ingredients synthesis. BMC Genom. 2018, 19, 978. [CrossRef] [PubMed]

72. Ge, Y.; Cheng, Z.; Si, X.; Ma, W.; Tan, L.; Zang, X.; Wu, B.; Xu, Z.; Wang, N.; Zhou, Z. Transcriptome profiling provides insight into the genes in carotenoid biosynthesis during the mesocarp and seed developmental stages of avocado (Persea americana). Int. J. Mol. Sci. 2019, 20, 4117. [CrossRef] [PubMed]

73. Jensen, S.R.; Franzyk, H.; Wallander, E. Chemotaxonomy of the Oleaceae: Iridoids as taxonomic markers. Phytochemistry 2002, 60, 213-231. [CrossRef]

74. Zhang, C.; Ma, X.; Zhu, C.; Chen, Z.; Li, W.; Zhao, X.; He, S.; Du, H. Analysis on the content of two chemical composition in different parts of cultivated and wild Centranthera grandiflora. Guihaia 2019, 1-7. [CrossRef]

75. Zhang, F.; Liu, W.; Xia, J.; Zeng, J.; Xiang, L.; Zhu, S.; Zheng, Q.; Xie, H.; Yang, C.; Chen, M. Molecular characterization of the 1-deoxy-D-xylulose 5-phosphate synthase gene family in Artemisia annua. Front. Plant Sci. 2018, 9, 952. [CrossRef]

76. Palazón, J.; Cusidó, R.M.; Bonfill, M.; Morales, C.; Piñol, M.T. Inhibition of paclitaxel and baccatin III accumulation by mevinolin and fosmidomycin in suspension cultures of Taxus baccata. J. Biotechnol. 2003, 101, 157-163. [CrossRef]

77. Grabkowska, R.; Mielicki, W.; Wielanek, M.; Wysokińska, H. Changes of phenylethanoid and iridoid glycoside distribution in various tissues of shoot cultures and regenerated plants of Harpagophytum procumbens (Burch.) DC. ex Meisn. S. Afr. J. Bot. 2014, 95, 159-164. [CrossRef]

78. Zhang, X.; Liu, C.J. Multifaceted regulations of gateway enzyme phenylalanine ammonia-lyase in the biosynthesis of phenylpropanoids. Mol. Plant 2015, 8, 17-27. [CrossRef]

79. Khakdan, F.; Alizadeh, H.; Ranjbar, M. Molecular cloning, functional characterization and expression of a drought inducible phenylalanine ammonia-lyase gene (ObPAL) from Ocimum basilicum L. Plant Physiol. Biochem. 2018, 130, 464-472. [CrossRef]

80. Xu, F.; Cai, R.; Cheng, S.; Du, H.; Wang, Y. Molecular cloning, characterization and expression of phenylalanine ammonia-lyase gene from Ginkgo biloba. Afr. J. Biotechnol. 2008, 7, 721-729. [CrossRef]

81. Song, J.; Wang, Z. Molecular cloning, expression and characterization of a phenylalanine ammonia-lyase gene (SmPAL1) from Salvia miltiorrhiza. Mol. Biol. Rep. 2009, 36, 939-952. [CrossRef] [PubMed]

82. Zhang, Y.; Fu, X.; Hao, X.; Zhang, L.; Wang, L.; Qian, H.; Zhao, J. Molecular cloning and promoter analysis of the specific salicylic acid biosynthetic pathway gene phenylalanine ammonia-lyase (AaPAL1) from Artemisia annua. Biotechnol. Appl. Biochem. 2016, 63, 514-524. [CrossRef] [PubMed]

83. Chang, A.; Lim, M.; Lee, S.; Robb, E.J.; Nazar, R.N. Tomato phenylalanine ammonia-lyase gene family, highly redundant but strongly underutilized. J. Biol. Chem. 2008, 283, 33591-33601. [CrossRef] [PubMed]

84. Kellogg, E.A. Different ways to be redundant. Nat Genet 2019, 51, 770-771. [CrossRef]

85. Lachowiec, J.; Mason, G.A.; Schultz, K.; Queitsch, C. Redundancy, feedback, and robustness in the Arabidopsis thaliana BZR/BEH gene family. Front. Genet. 2018, 9, 523. [CrossRef]

86. Whitaker, J.R. Polyphenol Oxidase. In Food Enzymes: Structure and Mechanism; Wong, D.W.S., Ed.; Springer: Boston, MA, USA, 1995; pp. 271-307. [CrossRef]

87. Taranto, F.; Pasqualone, A.; Mangini, G.; Tripodi, P.; Miazzi, M.; Pavan, S.; Montemurro, C. Polyphenol oxidases in crops: Biochemical, physiological and genetic aspects. Int. J. Mol. Sci. 2017, 18, 377. [CrossRef]

88. Tran, L.T.; Taylor, J.S.; Constabel, C.P. The polyphenol oxidase gene family in land plants: Lineage-specific duplication and expansion. BMC Genom. 2012, 13, 395. [CrossRef]

89. Vaughn, K.; Lax, A.; Duke, S. Polyphenol oxidase. Handb. Plant Cytochem. 1987, 1, 159-162. 
90. Verma, R.; Tapwal, A.; Kumar, D.; Puri, S. Assessment of Antimicrobial Potential and Phytochemical Profiling of Ethnomedicinal Plant Bergenia ciliata (haw.) sternb. in Western Himalaya. J. Microbiol. Biotechnol Food Sci. 2019, 9, 15-20. [CrossRef]

91. Sharma, S.K.; Patil, A.; Agnihotri, A.K.; Mehrotra, S. In vitro conservation of Alectra chitrakutensis: A critically endangered root parasitic plant of high medicinal importance. Acta Physiol. Plant. 2018, 40, 29. [CrossRef]

92. Michael, E.; Mohan, V.R. Determination of bioactive components of Caralluma umbellata haw. (apocynaceae) by gas chromatography and mass spectroscopy analysis. Asian J. Pharm. Clin. Res. 2018, 11, 194-199. [CrossRef]

93. Agrawal, P.; Laddha, K.; Tiwari, A. Isolation and HPLC method development of azafrin from Alectra parasitica var. chitrakutensis. Nat. Prod. Res. 2014, 28, 940-944. [CrossRef] [PubMed]

94. Paul, M. Medicinal Natural Products: A Biosynthetic Approach, 3rd ed.; John Wiley and Sons, Ltd.: West Sussex, UK, 2009; p. 303. [CrossRef]

95. Brocker, C.; Vasiliou, M.; Carpenter, S.; Carpenter, C.; Zhang, Y.; Wang, X.; Kotchoni, S.O.; Wood, A.J.; Kirch, H.; Kopečný, D. Aldehyde dehydrogenase (ALDH) superfamily in plants: Gene nomenclature and comparative genomics. Planta 2013, 237, 189-210. [CrossRef] [PubMed]

96. Cheng, X.; Zhao, X.; Huang, C.; Zhang, X.; Lyu, Y. Lutein content in petals and leaves of marigold and analysis of lutein synthesis gene expression. Acta Physiol. Plant. 2019, 41, 128. [CrossRef]

97. Hyun, T.K.; Rim, Y.; Jang, H.; Kim, C.H.; Park, J.; Kumar, R.; Lee, S.; Kim, B.C.; Bhak, J.; Nguyen-Quoc, B. De novo transcriptome sequencing of Momordica cochinchinensis to identify genes involved in the carotenoid biosynthesis. Plant Mol. Biol. 2012, 79, 413-427. [CrossRef]

98. Lin, H.; Wang, R.; Qian, Q.; Yan, M.; Meng, X.; Fu, Z.; Yan, C.; Jiang, B.; Su, Z.; Li, J. DWARF27, an iron-containing protein required for the biosynthesis of strigolactones, regulates rice tiller bud outgrowth. Plant Cell 2009, 21, 1512-1525. [CrossRef]

99. Abuauf, H.; Haider, I.; Jia, K.; Ablazov, A.; Mi, J.; Blilou, I.; Al-Babili, S. The Arabidopsis DWARF27 gene encodes an all-trans-/9-cis- $\beta$-carotene isomerase and is induced by auxin, abscisic acid and phosphate deficiency. Plant Sci. 2018, 277, 33-42. [CrossRef]

100. Vallabhaneni, R.; Bradbury, L.M.; Wurtzel, E.T. The carotenoid dioxygenase gene family in maize, sorghum, and rice. Arch. Biochem. Biophys. 2010, 504, 104-111. [CrossRef]

101. Zhang, X.; Allan, A.; Li, C.; Wang, Y.; Yao, Q. De novo assembly and characterization of the transcriptome of the Chinese medicinal herb, Gentiana rigescens. Int. J. Mol. Sci. 2015, 16, 11550-11573. [CrossRef]

102. Haas, B.J.; Papanicolaou, A.; Yassour, M.; Grabherr, M.; Blood, P.D.; Bowden, J.; Couger, M.B.; Eccles, D.; Li, B.; Lieber, M.; et al. De novo transcript sequence reconstruction from RNA-seq using the Trinity platform for reference generation and analysis. Nat. Protoc. 2013, 8, 1494-1512. [CrossRef] [PubMed]

103. Davidson, N.M.; Oshlack, A. Corset: Enabling differential gene expression analysis for de novoassembled transcriptomes. Genome Biol. 2014, 15, 410. [CrossRef] [PubMed]

104. Paulino, P.R.; Diego Mauricio, R.O.P.; Corrêa, L.G.G.; Rensing, S.A.; Birgit, K.; Bernd, M.R. PlnTFDB: Updated content and new features of the plant transcription factor database. Nucleic Acids Res. 2010, 38, D822-D827. [CrossRef]

105. Young, M.D.; Wakefield, M.J.; Smyth, G.K.; Oshlack, A. Method Gene ontology analysis for RNA-seq: Accounting for selection bias. Genome Biol. 2010, 11, R14. [CrossRef] [PubMed]

106. Mao, X.; Cai, T.; Olyarchuk, J.G.; Wei, L. Automated genome annotation and pathway identification using the KEGG Orthology (KO) as a controlled vocabulary. Bioinformatics 2005, 21, 3787-3793. [CrossRef] [PubMed]

(C) 2019 by the authors. Licensee MDPI, Basel, Switzerland. This article is an open access article distributed under the terms and conditions of the Creative Commons Attribution (CC BY) license (http://creativecommons.org/licenses/by/4.0/). 